\title{
From 1.5 Meters to 10.4 Meters in 20 Years: Technological Evolution at the Instituto de Astrofísica de Canarias in the Development of Infrared Instrumentation for Terrestrial Telescopes
}

\author{
P. G. R. Caicoya* ${ }^{\ddagger}$, M. B. Cabrera ${ }^{\dagger, \S}$, P. F. Izquierdo*, ${ }^{*}, J^{*}$ Patrón $^{\dagger, \|}$, \\ V. Sánchez De La Rosa*,** and F. T. Sangines, ${ }^{*} \dagger \dagger$ \\ * Mechanical Department, Instituto de Astrofísica de Canarias (IAC) \\ San Cristóbal de la Laguna, Canarias, España \\ ${ }^{\dagger}$ Management Department, IAC \\ San Cristóbal de la Laguna, Canarias, España \\ ${ }_{\ddagger}^{\ddagger}$ predondo@iac.es \\ $\S$ mbarreto@iac.es \\ ๆpfi@iac.es \\ 1|jpr@iac.es \\ **vsr@iac.es \\ $\dagger^{\dagger}$ ftenegi@iac.es
}

Received 2013 June 28; Revised 2014 April 9; Accepted 2014 May 7; Published 2014 June 13

\begin{abstract}
This paper reviews the evolution of the technical development of infrared instrumentation for terrestrial telescopes at the Instituto de Astrofísica de Canarias (IAC). The three most representative instruments, CAIN (installed on the $1.5 \mathrm{~m}$ Carlos Sánchez Telescope), LIRIS (on the $4.2 \mathrm{~m}$ William Herschel Telescope), and EMIR (on the $10.4 \mathrm{~m}$ Gran Telescopio Canarias) are described and compared. Each project entailed an improvement and a technological advance in relation to its predecessor. Larger optical beams brought larger lenses, larger mechanisms to hold them, stiffer and more massive support structures, and a scale factor upgrade of their cryostats. The evolution of infrared projects has been closely linked to the development of the manufacturing, assembly, integration and verification facilities at the IAC and has also had a strong influence on management techniques. As an indication of the growth in complexity of projects over time, this paper highlights the framework of available human resources, established consortia, timing and costs associated with the three instruments.
\end{abstract}

Keywords: Infrared, instrumentation, cryogenic, EMIR.

\section{Introduction: The Evolution of the Telescopes and Infrared Instruments}

This paper focuses on the developments of main infrared (IR) instruments (CAIN, LIRIS, and EMIR) of the Instituto de Astrofísica de Canarias (IAC) as a showcase of the evolution at the institution in the complex technological field of cryogenic instrumentation.

After a brief introduction of the telescopes and the basic layouts of the instruments, an insight into their mechanics (cryostats, support structures, lens mounts and mechanisms) is followed by a description of infrastructure at the IAC, and finally the management of the project teams is discussed.

\subsection{The telescopes}

\subsubsection{Carlos Sánchez telescope}

Infrared observations in the Canary Islands were made at the Observatorio del Teide (Tenerife) in the early '70s, when the Department of Astrophysics at Imperial College of Science, Technology and Medicine (ICSTM, UK) decided to build and install a $1.5 \mathrm{~m}$ infrared telescope (the largest infrared telescope at that time) at the observatory. 
The $1.5 \mathrm{~m}$ Infrared Flux Collector, later renamed the Carlos Sánchez Telescope (TCS), in memory of the pioneer of the infrared astrophysics in Spain, was proposed, designed and constructed under the direction of Professor Jim Ring (Ring, 1968; Selby \& Mampaso, 1991; for a detailed description of the telescope see Barlow, 1975, pp. 163-164 and Jones, 1978), in collaboration with other groups from the United Kingdom and the IAC. The IAC's then Director, Professor Francisco Sánchez, assigned Professor Carlos Sánchez to join the English team to learn and lay the groundwork for infrared astrophysics in Spain.

The TCS was originally conceived as an inexpensive "flux bucket" with very relaxed specifications. The success of this telescope, which has been operative since 1972, made it the prototype for the $3.8 \mathrm{~m}$ UK Infrared Telescope (UKIRT). The design also altered the traditional proportions of telescopes and their domes: the dome for the TCS cleared the telescope by just a few inches. Previous designs had domes proportionally much larger than the telescopes they housed.

Ring's most influential work was the implementation of thin mirror telescopes. At the beginning of his career it was usual for telescope primary mirrors to have a thickness-to-diameter ratio of 1:5 or 1:4, i.e. the mirror worked as a rigid solid. The telescope built by Ring has a ratio of about 1:12, making the TCS one of the first thin mirror telescopes.

The TCS's main mirror is carried on an equatorial mount with an $f / 13.8$ Cassegrain focus in a Dall-Kirkham configuration. It has an effective focal length of $21 \mathrm{~m}$. The TCS has been the property of the IAC since 1982, when it was transferred by the then SERC (Science and Engineering Research Council, UK), its original owner. Since 1987 the mechanics and electronics of the telescope have been improved on a continuous basis.

\subsubsection{William Herschel telescope}

The William Herschel Telescope (WHT) is the largest optical telescope with a monolithic mirror in Europe, with a $\varnothing 4.2 \mathrm{~m}$ primary mirror. The WHT is part of the Isaac Newton Group of Telescopes (ING), which also operates the Isaac Newton Telescope and the Jacobus Kapteyn Telescope. The ING is owned and operated jointly by the Particle Physics and Astronomy Research Council (PPARC, UK), the Nederlandse Organisatie voor Wetenschappelijk Onderzoek (NWO, Netherlands) and the IAC.

The WHT (Sanchez \& Wall, 1991) is a generalpurpose facility, instrumented to allow a wide range of astronomical observations, from the optical to the infrared, covering both imaging and spectroscopy. The WHT's conception dates back to the late 1960s, when astronomers were wondering what kind of powerful telescope they should build in the northern hemisphere. The SERC started to plan the WHT in 1974. The precise diameter of $4.2 \mathrm{~m}$ was determined by the availability of a mirror blank of that size, made by Owens-Illinois (USA). The mirror was finished at Grubb Parsons (UK). It is believed that this was the most accurate large mirror ever made until that time. Construction of the telescope enclosure began in 1983 and first light took place on June 1, 1987.
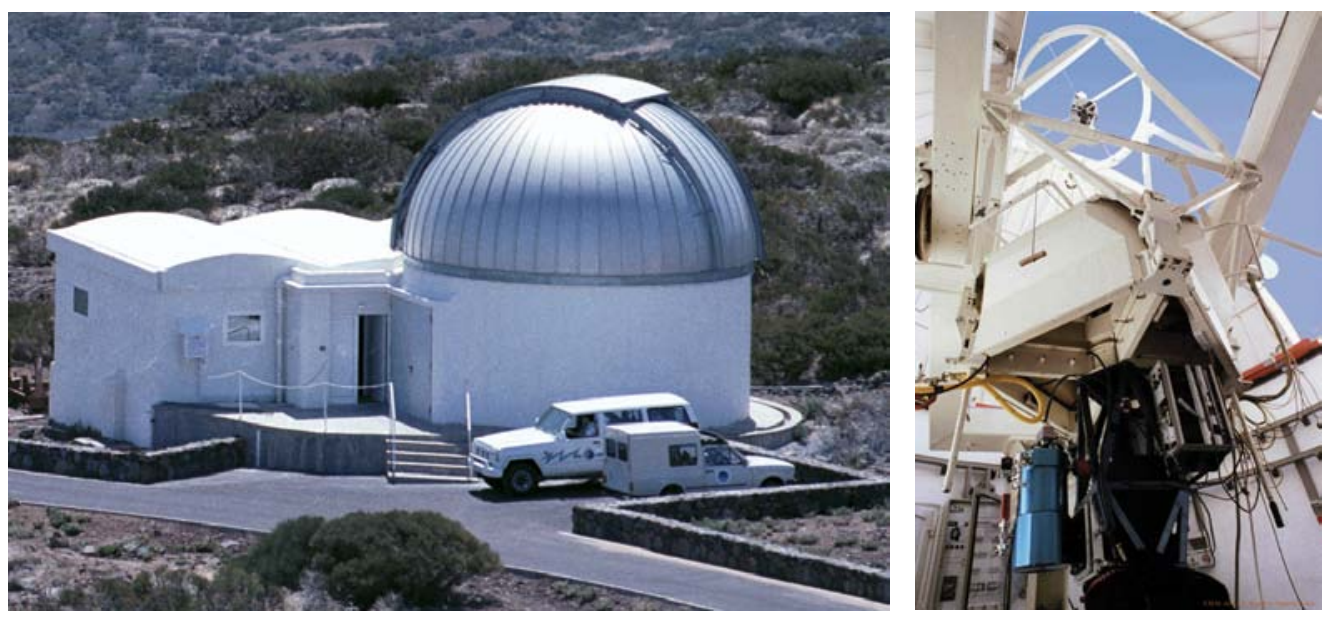

Fig. 1. The Carlos Sánchez Telescope in the Observatorio del Teide (Tenerife, Spain). 

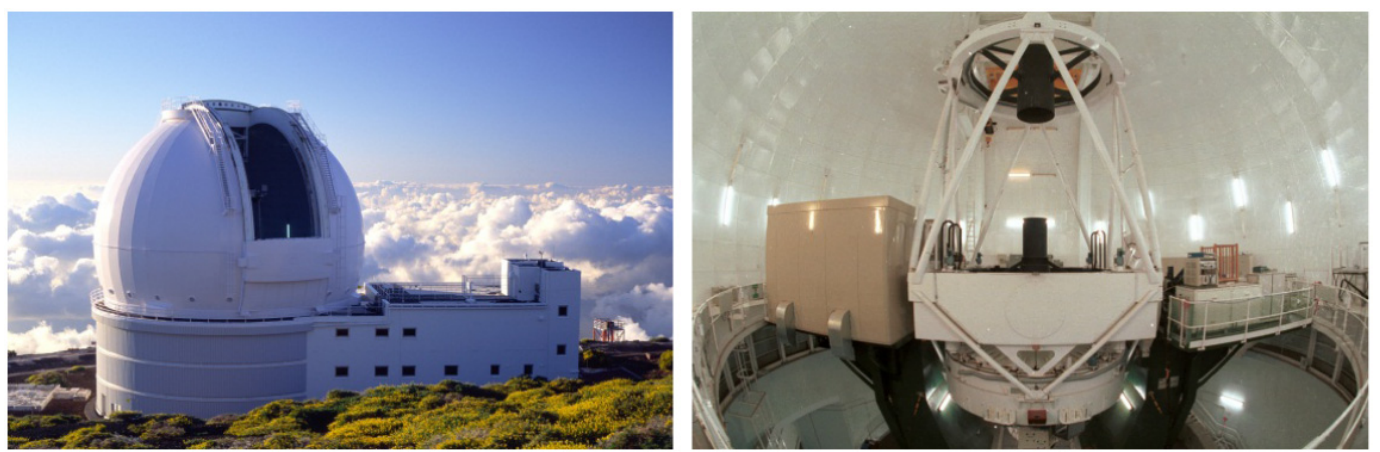

Fig. 2. The William Herschel Telescope at the Observatorio del Roque de Los Muchachos (La Palma, Spain).

The WHT has a classical Cassegrain configuration, with a paraboloidal primary mirror made of a glass-ceramic, Cervit, with nearly zero coefficient of thermal expansion, and coated with a thin layer of aluminum. The effective focal ratio of the primary mirror is $f / 2.8$, and the unvignetted field of view is $40 \mathrm{arcmin}$. When not operating at prime focus, a convex hyperboloidal secondary mirror, made of Zerodur, $1.0 \mathrm{~m}$ in diameter, directs the light through a central hole in the primary mirror to the main instrumentation mounted at the Cassegrain focus beneath the primary mirror cell. The telescope also incorporates a flat tertiary mirror, angled at $45^{\circ}$, so that the light from the secondary is reflected sideways through one of the two altitude bearings to the Nasmyth platforms, where heavy instruments can be mounted.

The effective focal length of the telescope for the Cassegrain and Nasmyth foci is $46.2 \mathrm{~m}(f / 11)$. The WHT mounting has an alt-azimuth design. The mass of the telescope's moving structure is around 200 metric tons.

\subsubsection{Gran telescopio de Canarias}

In the mid 1980s the IAC took on the great challenge of developing and building a large telescope for its observatories. This development began with a proposal for a monolitic $8 \mathrm{~m}$ class telescope (Álvarez Martin et al., 1995), which finally evolved into the $10.4 \mathrm{~m}$ segmented primary mirror, the Gran Telescopio Canarias (GTC; Álvarez Martín et al., 1997), the largest optical and infrared telescope in the world to date. Construction work began at the site in 2000 at the Observatorio del Roque de los Muchachos, La Palma, and first light took place on July 14, 2007. The telescope has been operational since March 2009.

The GTC's primary mirror consists of 36 hexagonal segments that together act as a single

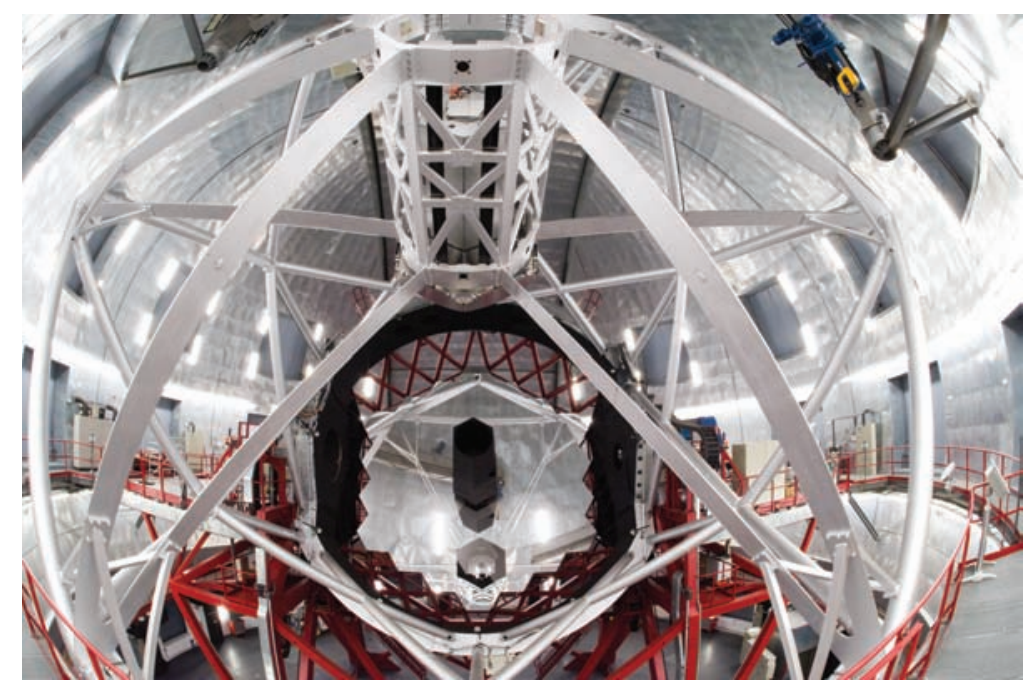

Fig. 3. The Gran Telescopio Canarias, the largest optical and infrared telescope in the world to date. 


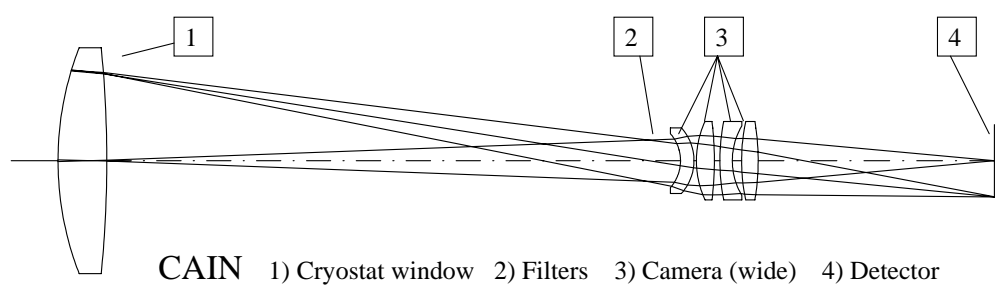

Fig. 4. The optical layout of CAIN.

mirror. The light-collecting mirror surface area of the GTC is equivalent to a $10.4 \mathrm{~m}$ diameter single monolithic mirror and has $73 \mathrm{~m}^{2}$ of effective area. The GTC also has a secondary and a tertiary mirrors that, together with the primary mirror, produce the telescope focal plane in the focal station of choice. The telescope mount, a large steel structure that holds the mirrors cells, allows rotational movements of the telescope along a horizontal and vertical axis. The GTC has a classical Ritchey-Chrétien optical configuration, with the aperture in the secondary mirror to control the telescope emissivity in the infrared range. It has an effective focal length of $170 \mathrm{~m}$, a plate scale of $0.21 \mathrm{arcsec} \cdot \mathrm{mm}^{-1}$ and fields of view of $20 \mathrm{arcmin}$ at the Nasmyth focus, 15 arcmin at the Cassegrain focus and 5 arcmin at the folded Cassegrain focus. The GTC will be the last of the generation of so called 8-10 m class telescopes. Efforts have therefore been made to improve its design over that of its predecessors and to learn from earlier experience. Thanks to its huge collecting area and advanced engineering, the GTC is classed among the bestperforming telescopes for astronomical research. The total telescope moving mass is nearly 400 metric tons.

\subsection{The infrared instruments}

CAIN (Cámara Infrarroja), LIRIS (Long Slit Intermediate Resolution Infrared Spectrograph) and EMIR (Espectrógrafo Multiobjeto InfraRrojo) share the basic layout of a typical infrared camera (see Fig. 4): light coming from the telescope enters the cryostat through a window that acts as a field lens; a camera then focuses the beam onto the detector surface; and, before or after that camera, a filter wheel allows selection of the desired wavelengths. The initial concept of CAIN included only one camera, but finally both a wide field and a

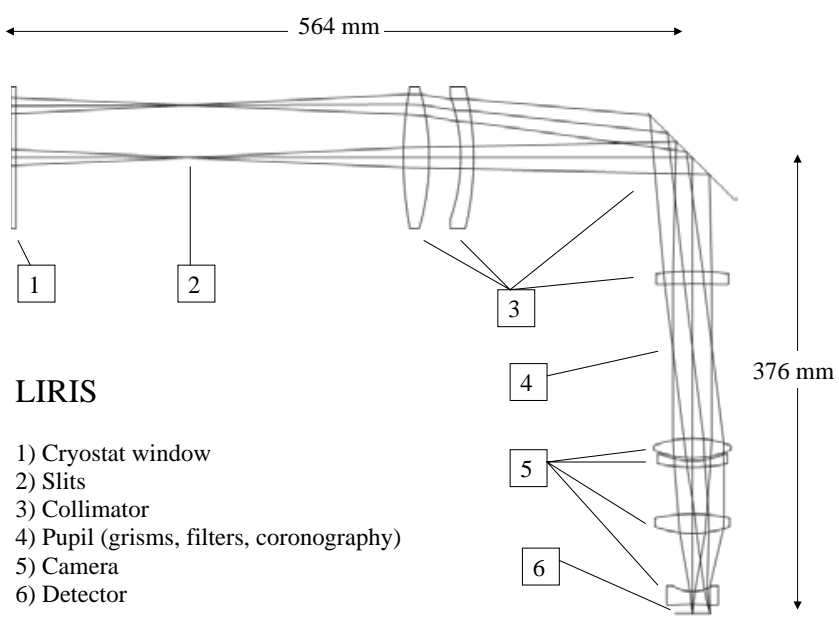

Fig. 5. The optical layout of LIRIS.

narrow field camera were mounted on a remotely operated wheel.

LIRIS (see Fig. 5) takes two leaps forward: the size of the $4.2 \mathrm{~m}$ WHT is almost three times that of the $1.5 \mathrm{~m}$ TCS, moreover, spectrographic capabilities are added to the basic camera layout. Multiple slit masks are implemented to isolate the targets whose light spectrum is being studied on an extra wheel, the slit wheel. In order to reduce the envelope of the instrument, a flat mirror is used to fold the beam. Several dispersive elements, grisms, are mounted on a four-motor wheel module that carries filters, grisms, pupils, and coronographic masks. In the interest of optical simplicity, the beam is collimated at the entrance of these grisms: this set-up requires extra lenses, the collimator lenses, installed before the grism in the optical path. The camera is mounted on a wheel that allows the user to replace it remotely by a free aperture. A focal stage translation unit was designed to displace the detector to optimize the focusing of the beam on its surface, but this unit was not finally installed.

LIRIS's mechanical conceptual design was subcontracted to the Royal Observatory, Edinburgh 


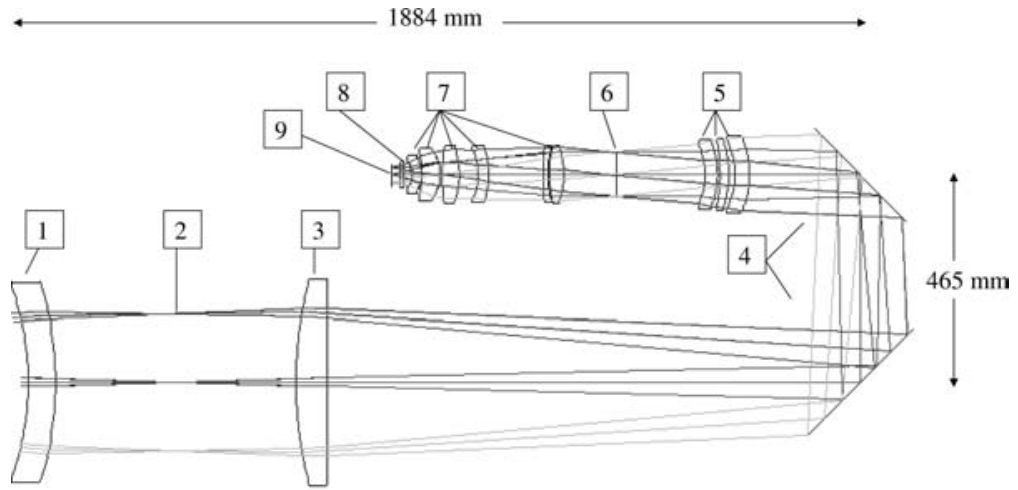

EMIR 1) Cryostat window 2) Slits 3) Collimator \#1 4) Periscope

$\begin{array}{lllll}5 \text { 5) Collimator \#2 6) Pupil (grisms) 7) Camera } & \text { 8) Filters } & \text { 9) Detector }\end{array}$

Fig. 6. The optical layout of EMIR.
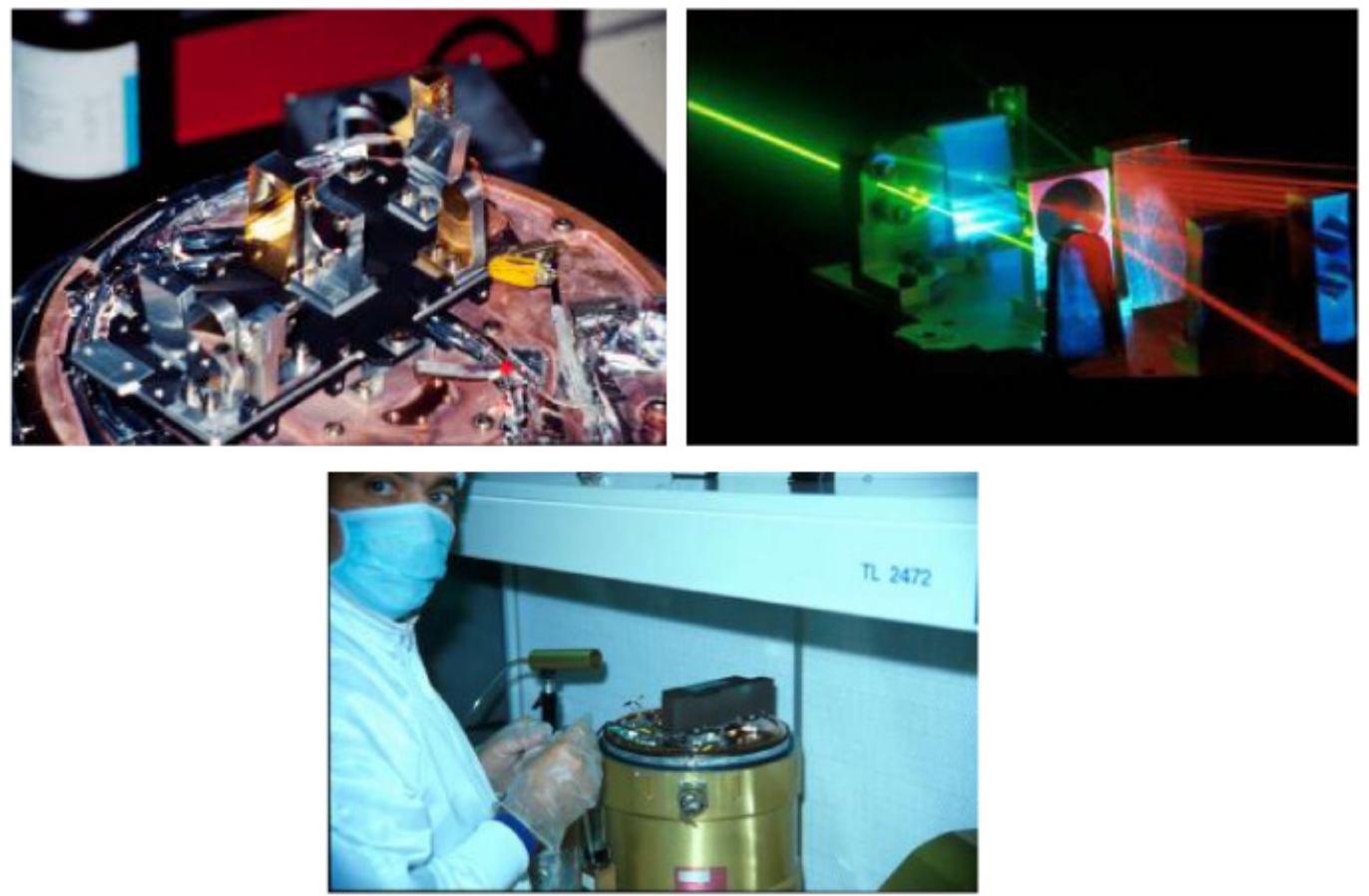

Fig. 7. ISOPHOT-S AIV stage at the IAC.

(ROE, UK), and the detailed design was performed at IAC.

EMIR, again, takes the "just learned" technology another two steps further (see Fig. 6). First, it is the largest and most complex instrument on the GTC. Second, it is state of the art: instead of discrete slit masks that must be replaced, a configurable slit unit will allow for remote reconfiguration of the slit pattern generating up to 55 simultaneous slits.

The beam is folded twice to reduce the physical envelope of the cold mass and the detector is mounted on a motorized mechanism in tripod configuration, which provides both continuous compensation of the mechanical flexure of the instrument during instrument rotation and focusing capabilities.

Although the development of the three main infrared instruments has been the main source of the IAC's expertise in this field of cryogenic instrumentation, we must not fail to mention other projects that also incorporate cryogenic technology, such as the ISOPHOT-S spectrometer for the ISO satellite, which was the first infrared instrument in 
which the IAC was involved; the now decommissioned infrared photometers CVF and FIN for the TCS; the Tenerife Infrared Polarimeters, TIP I \& II for the VTT and Gregor solar telescopes; FRIDA and MIRADAS for the GTC; the Multifrequency Instrument, MFI, for the QUIJOTE CMB experiment; and HARMONI, one of the first instruments selected for the E-ELT, which is set to inherit all the know-how described in this report.

\section{Mechanical Systems}

This section summarizes, from the outside, as evident to the non-expert, to the inside, more hidden from the eye, how mechanical systems have evolved from CAIN to EMIR.

\subsection{Cryostats}

Infrared observations depend on the use of cryogenic and vacuum enclosures to allow the instruments to efficiently manage the faint IR radiation captured from the sky. The required extremely cold, highly evacuated environments (typically at $-196^{\circ} \mathrm{C}$ and $10^{-6}$ mbar) are achieved inside vacuum chambers equipped with cooling systems, commonly referred to as cryostats. The IAC has considerable experience in the use and design of cryostats, and also some experience in manufacturing small cryostats. Besides CAIN, LIRIS, and EMIR, a fourth cryostat is added to the list in this chapter, the EMCTS (EMIR Multipurpose Cryogenic Tests System), the largest test cryostat designed and used at the IAC to date.

\subsubsection{CAIN}

An ND-5 customized cryostat (see Fig. 8) from Infrared Laboratories (USA) has been used for the CAIN instrument. It consists of a dewar containing a single cryogenic vessel with a cooled radiation shield surrounding the vessel and the cold working surface. The opto-mechanical components are attached directly to the cold base of the cryostat, with a diameter of $130 \mathrm{~mm}$ and a height of $38 \mathrm{~mm}$. The main features of this cryostat are an outer diameter of $177 \mathrm{~mm}$, a total mass of $11 \mathrm{~kg}$, and an inner volume for the coolant (liquid nitrogen, $\mathrm{LN}_{2}$ ) of 4.9 liters.

\subsubsection{LIRIS}

LIRIS cryostat (Fig. 9) is a welded cylindrical vessel. It is made of AISI 304L stainless steel, in three sections: a central ring and two end covers. The front cover is a circular plate which carries the window and its mount and a cover over the port giving access to the entrance wheel. It is not necessary to remove the front cover to change focal plane masks (Fig. 10).

The rear cover can be removed to gain access to all the other internal modules of the instrument and comprises a torospherical dome (the first designed at the IAC for a vacuum chamber). It has an auxiliary window to be used on the integration and alignment stages prior to the installation of the detector. The center section carries all the semi-permanent access ports for electrical wiring, cooling and vacuum pumping. Several Finite Elements Analyses (FEA) were carried out in order to
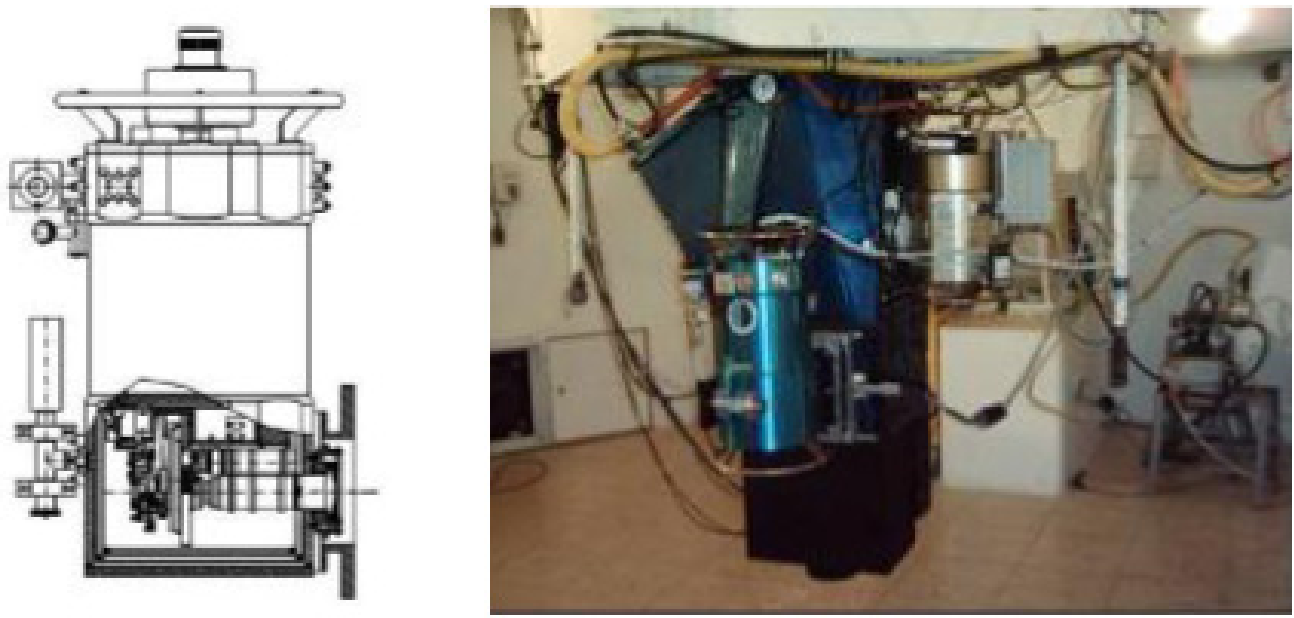

Fig. 8. CAIN cryostat (in blue, right image) installed on the TCS. 


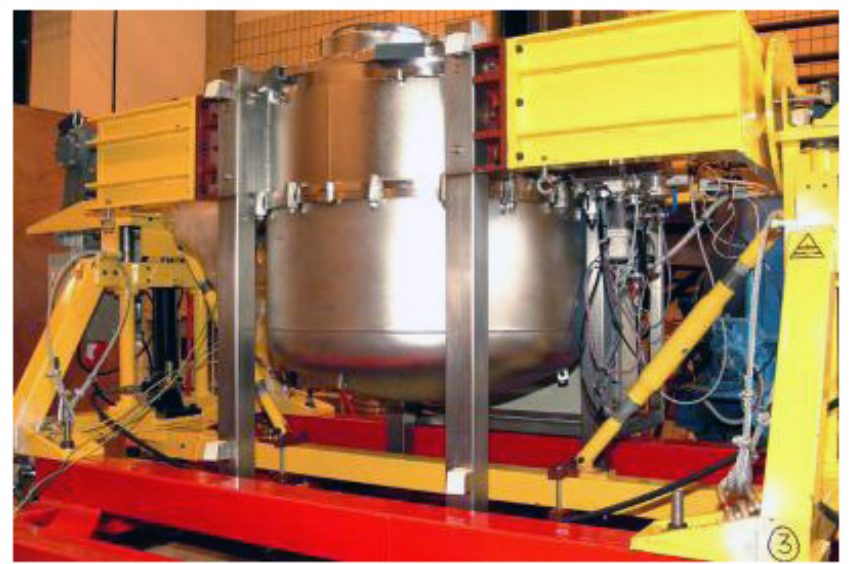

Fig. 9. LIRIS cryostat about to be delivered to the WHT.

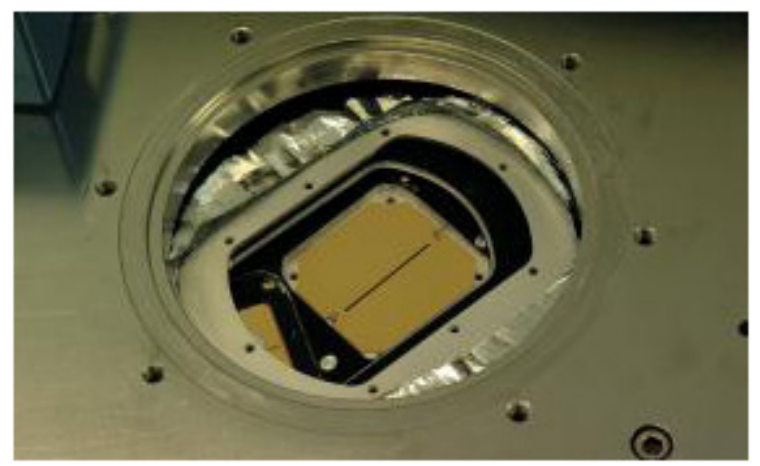

Fig. 10. LIRIS focal plane masks access.

optimize the design and to select a suitable material for the vacuum vessel.

\subsubsection{EMCTS}

The EMCTS (Fig. 11) is a cryostat designed to perform complete functional tests on large IR instrument subsystems, such as optical assemblies and mechanisms. This cryostat is adapted to the specific needs of EMIR main subsystems. The most demanding is the Configurable Slit Unit (CSU, a mechanism with a global envelope of $1200 \times 410 \times$ $70 \mathrm{~mm}^{3}$ ). The main geometry of the EMCTS is a cylinder of around $1 \mathrm{~m}$ diameter, $1 \mathrm{~m}$ long, and it has been equipped with two lateral extensions to accommodate the length of the CSU.

The design of the EMCTS was performed entirely by the EMIR engineering team at the IAC, and the manufacturing was contracted to the Spanish company Telstar Tecnología Mecánica in June 2003. The material of the vacuum chamber is stainless steel.

This cryostat uses an $\mathrm{LN}_{2}$ tank for pre-cooling, and then, a Close Cycle Cooler (CCC) system to maintain the temperature at the required values, around $77 \mathrm{~K}$. The CCC system consists of two Oerlikon-Leybold Vacuum COOLPOWER 5/100 cold heads fed by a COOLPOWER 6000D dual compressor, water refrigerated.

One of the particularities of this testing facility is that it has been mounted in a motorized rotating frame, which allows for functional testing of subsystems at any orientation with respect to gravity. This aspect covers all the possible structural configurations of EMIR subsystems during operation, since the instrument will be mounted at the Nasmyth rotator of the GTC telescope.

\subsubsection{EMIR}

The EMIR cryostat is the largest ever managed by the IAC. The final main geometry of the cryostat is a cylinder of $1.8 \mathrm{~m}$ diameter and $2.2 \mathrm{~m}$ length, i.e. a vacuum volume of about $5.5 \mathrm{~m}^{3}$, a factor 3 larger than the LIRIS or EMCTS cryostats.

In general, the design of the EMIR instrument has profited from most of the technical solutions adopted for the LIRIS instrument, as most of the EMIR engineering team members have participated partially or totally in development of LIRIS. In particular, the design of the EMIR cryostat has followed the proven solutions of both the LIRIS and the EMCTS cryostats, and has, again, been entirely developed by IAC mechanical engineers. Due to weight limitations for instruments to be mounted on the GTC, the vacuum chamber is made of aluminum alloy. The construction of the vacuum chamber was contracted to the Spanish company TRINOS Vacuum-Projects, which has considerable experience in the manufacture of cryogenic facilities and has very reliable aluminum welding procedures: part of the technical and management teams of this company are the same as for the EMCTS cryostat (one member also participated in the LIRIS cryostat), but the structure, organization, and name of the company has changed with time.

In order to cool EMIR down to the required operational levels, $77 \mathrm{~K}$ for the detector, and nearly $100 \mathrm{~K}$ for the other subsystems, this cryostat is equipped with two $\mathrm{LN}_{2}$ tanks for pre-cooling, each with a capacity of 33 liters. A CCC system is then used to maintain the cryogenic temperatures (four Oerlikon-Leybold Vacuum COOLPOWER 5/100 helium cold heads fed by two COOLPOWER 6000D dual compressors). 

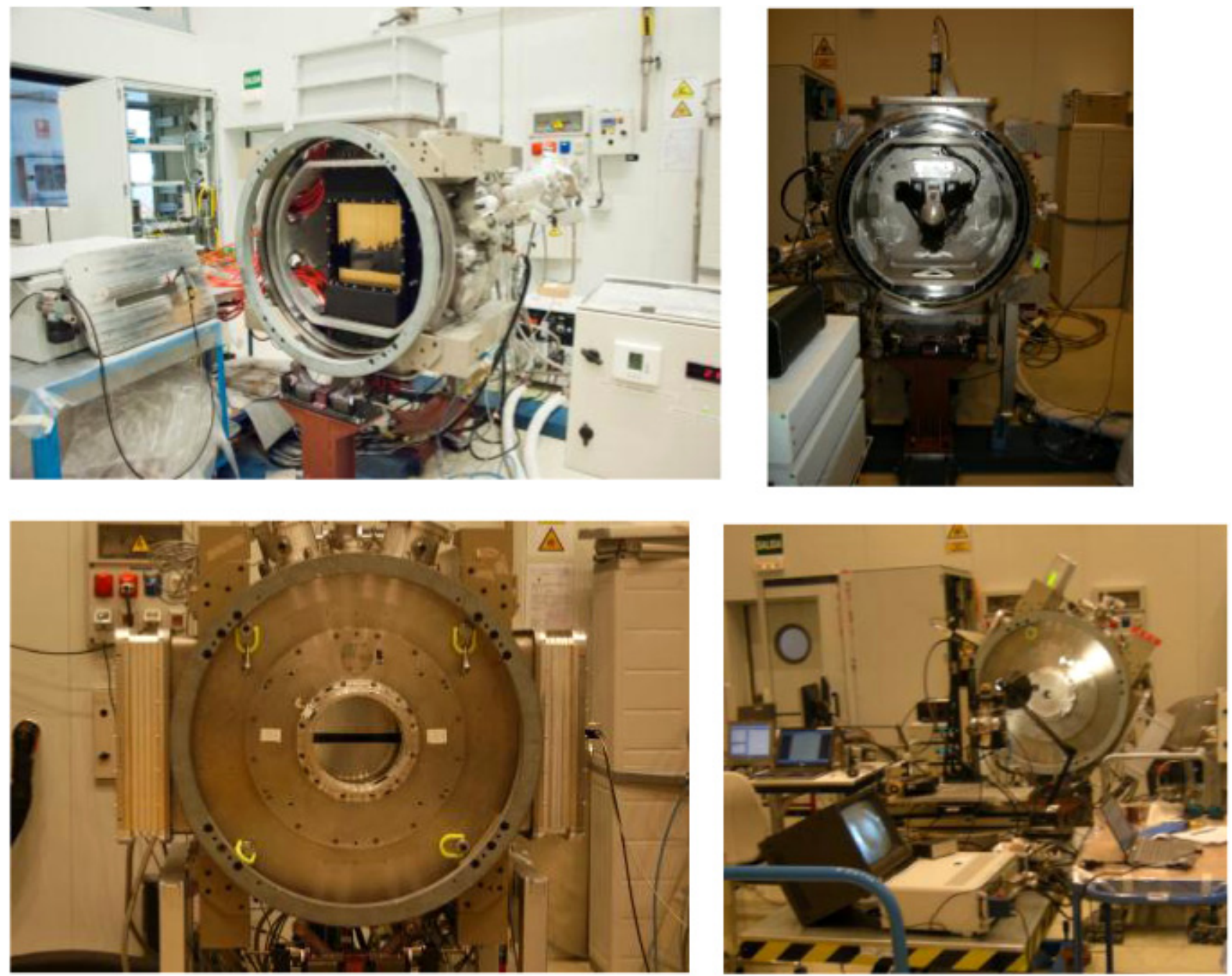

Fig. 11. The EMIR Multipurpose Cryogenic Test System.

For testing purposes during assembly, integration and verification (AIV) phases the cryostat is mounted in a motorized holding structure, which allows all the orientations of the instrument to be reproduced during normal operations once installed on the GTC Nasmyth platform and rotator. The rotator system can be programmed for any given orientation for systematic tests. The same structure is also used for holding the instrument during transportation (see Fig. 12).

The following table shows the main parameters for the cryostats discussed above.

\subsection{Support structures}

One of the most critical aspects to be solved in the design of the structure of astronomical instruments

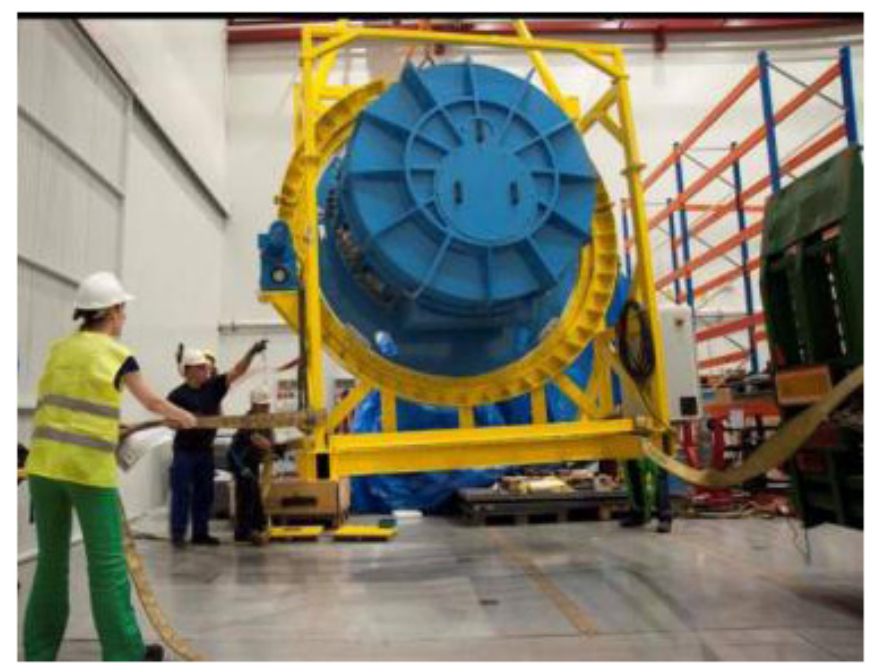

Fig. 12. EMIR cryostat on the AIV and transport structure. 
Table 1. Main characteristics of cryostats developed at the IAC for IR instrumentation.

\begin{tabular}{lclll}
\hline Name & Cryostat weight $(\mathrm{kg})$ & Cryostat size $(\mathrm{mm})$ & \multicolumn{1}{c}{ Cooling system } & Manufacturer \\
\hline CAIN & 11 & $\varnothing 177 \times 279$ & $\mathrm{LN}_{2}$ tank $+\mathrm{SN}_{2}$ tank & Infrared laboratories \\
LIRIS & 600 & $\varnothing 915 \times 900$ & $\mathrm{LN}_{2}$ pre-cooling tank $+\mathrm{CCC}$ & Ingovi \\
EMCTS & 1,100 & $\varnothing 1,000 \times 1,066$ & $\mathrm{LN}_{2}$ pre-cooling tank $+\mathrm{CCC}(2)$ & Telstar tecnología mecánica \\
EMIR & 1,700 & $\varnothing 1,800 \times 2,200$ & $\mathrm{LN}_{2}$ pre-cooling tanks $(2)+\mathrm{CCC}(4)$ & Trinos-Vacuum projects \\
\hline
\end{tabular}

is to minimize the motion of the opto-mechanical components and the detector, taking into consideration not only the flexure of the optical mounts themselves but also that of the mechanical interface with the telescope (the movement of the instrument occurs during operation, inducing changes in orientation with respect to the gravity vector). These requirements can be achieved following a suitable design process, and then using flexure compensation (active or passive) of the instrument.

While CAIN's structure was just the cold base of an off-the-shelf cryostat (see Fig. 13), the detailed design of the support structure of LIRIS, including all the calculations and verifications, was done at the IAC, and the manufacturing process was subcontracted to the Ingovi company. Two main subsystems form the support structure, the optical bench and the support trusses; this structure is the

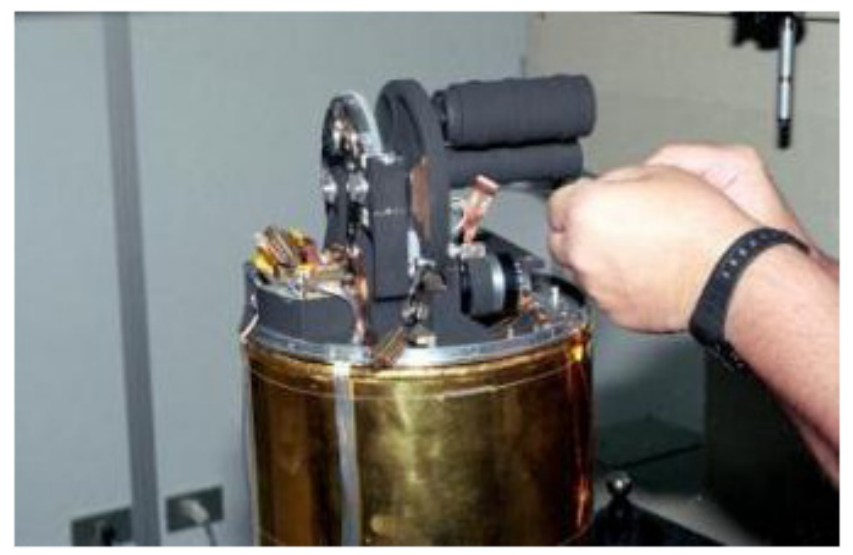

Fig. 13. CAIN support structure.
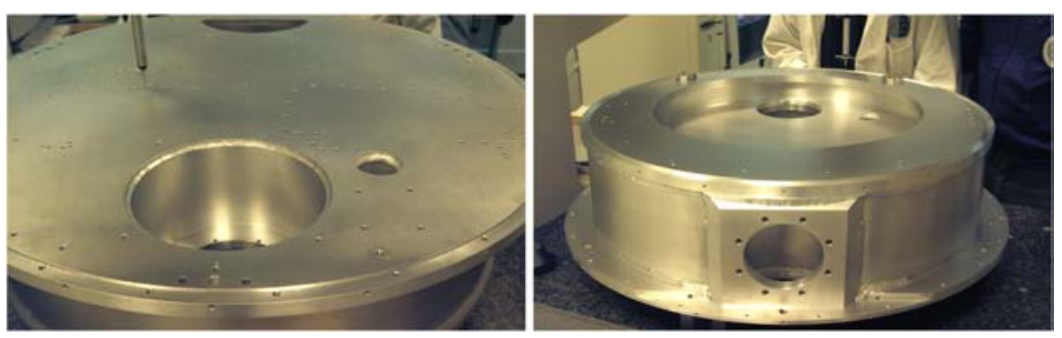

Fig. 14. LIRIS support structure. largest (with a diameter of $750 \mathrm{~mm}$ and a depth of $185 \mathrm{~mm}$ ) and heaviest $(65 \mathrm{~kg}$ ) of the cooled components in the instrument. The optical bench $(\mathrm{OB})$ is a conventional TIG welded structure, made of aluminum alloy 6061-T6 plates, and supported from the central section of the vacuum vessel by three $\mathrm{V}$ shaped G-10 glass-epoxy composite trusses. The OB consists of a front plate (supporting the entrance wheel) and a rear plate (thicker and used as a reference surface and mounting plate for all the other opto-mechanical cold components) joined by seven shear webs, three tubes (enclosing the collimator, access to the second stage of the CCC head, and a wiring passage) and an outer ring that forms a continuous seal and permits the use of the interior of the bench as a tank for $\mathrm{LN}_{2}$. The three $\mathrm{V}$-shaped trusses are machined from G-10 glass-epoxy composite, and as the OB contracts on cooling, they bend out of plane applying moments to the edge of the bench.

The change in scale (in size, weight, and complexity) from LIRIS to EMIR brought about the necessity for a large cryostat to test the optomechanical components and mechanisms of the latter instrument. In this scenario EMCTS serves as a tool to guarantee the final performance of the subsystems and the whole instrument. From a design point of view, the EMCTS is a medium complexity system, considering that it is not an "instrument" in the strict sense, so image quality is not a requirement. In any case, a very careful calculation and design process was undertaken. In the case of LIRIS, the support structure consists 
of an optical bench and three support trusses. The OB is the main cold component of the EMCTS and is basically a hollow cylindrical structure made of TIG - welded aluminum alloy 6082-T6 plates. The main functions of the OB are to support all EMIR's opto-mechanical components and mechanisms to be tested. As in the case of LIRIS, the OB has a front plate (reference surface and attaching area for the subsystems to be tested), a rear plate (to connect the thermal links of the CCCs), seven stiffeners (to facilitate the thermal conduction and to permit the system to withstand both vacuum and over-pressure conditions), and an outer ring that constitutes the continuous sealing between the two main plates (to allow the use of the $\mathrm{OB}$ as an $\mathrm{LN}_{2}$ tank). The main functions of the support trusses are to hold the structures and to position the $\mathrm{OB}$ relative to the Vacuum Chamber (VC), and to decouple them thermally to permit the differential contraction of the bench relative to the chamber, acting as the interface between both elements. They are manufactured from glass-epoxy composite G-10B in the central part and stainless steel 304-L at the tips in order to facilitate the attachment to the $\mathrm{OB}$ and the $\mathrm{VC}$.

The EMIR component blueprints were drawn up and the instrument designed to meet strict scientific requirements. From the support structure point of view, a method for the prediction for the optical components flexure was developed. This method takes into account the fact that the design of ground-based astronomical instruments is governed by tight restrictions on the displacements of

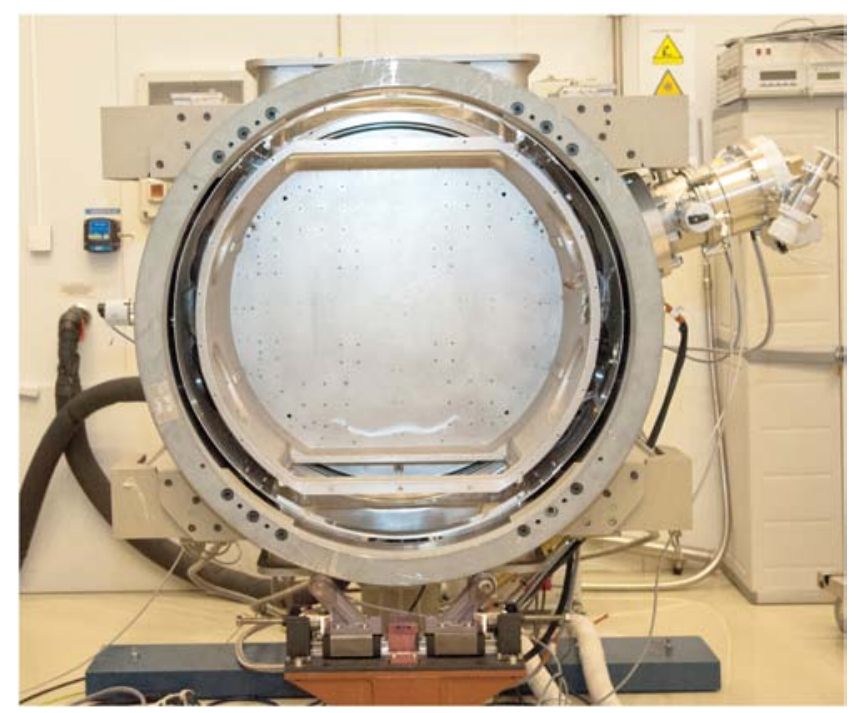

Fig. 15. EMCTS support structure. its optical components under thermal and gravity loads. Image stability during the scientific data acquisition process is one of the key parameters that determine the structural design of the new generation of astronomical instruments. The image stability prediction process of an optical instrument, in either the visible or the infrared, starts by computing using FEA the displacements and tilts of the support structure, induced by gravity and thermal loads. These displacements and tilts are later transferred to the optical model to obtain the image movements as a function of the spatial orientation and temperature of the instrument. Analysis of these results allows criteria for the structure optimization to be generated that will be validated in a new computation loop. The prediction process of the deflection on the optical components mounted on the support structure consists of three steps: image stability requirement calculations, error budget calculations, and the re-design loop. As guidelines for the design of the EMIR Optical Bench, the following aspects have been taken into account: stiffness, mass optimization, limitation of the maximum stress, good thermal conductivity, minimization of the differential thermal contractions, accessibility, and manufacturability. The FEA calculations of the assembly OB plus support trusses (the support structure of the instrument) included a full detailed study of the structural static behavior, eigen-frequencies, thermo-structural coupled behavior, sensitivity to variations of the center of gravity, the handling process, etc.

The EMIR optical bench has an H-shaped profile, made of thick plates $(50$ and $70 \mathrm{~mm})$ of aluminum alloy 6082-T6 joined through electron beam welding (EBW); this welding system minimizes the amount of heat supplied to the plates during the process, which affects the mechanical and thermal properties of the base material. The central flat plate provides stiffness in one direction, and two perpendicular flat plates provide stiffness and support to several optical components, minimizing the distance between their interfaces and the exterior of the cryostat, and improving accessibility during the integration and maintenance phases. The circular plate, located near the gravity center of the cold mass, constitutes the supported area in which the support trusses (radial as well as axial) are linked with the vacuum chamber. The manufacture of the OB was subcontracted to Trinos Vacuum Projects. 


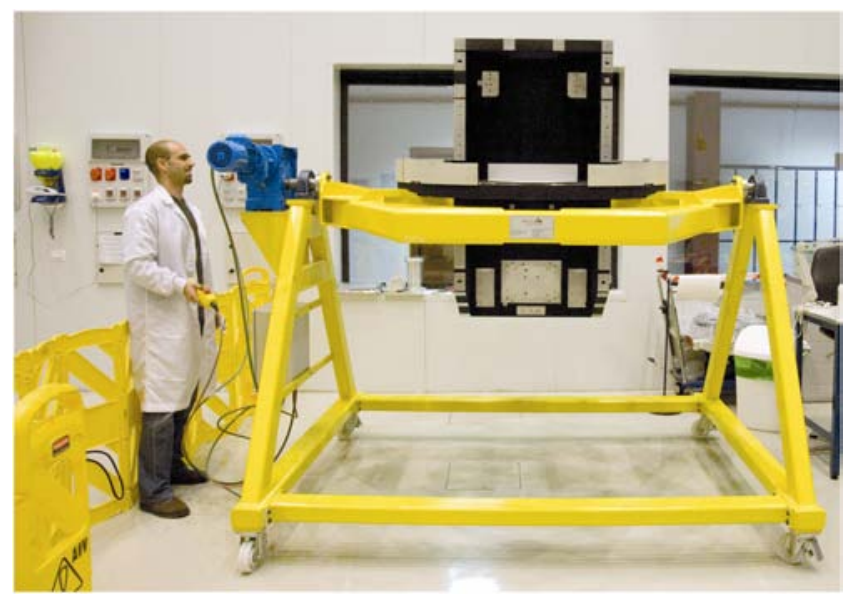

Fig. 16. EMIR optical bench.

Table 2. Comparative table of masses and sizes of the different support structures.

\begin{tabular}{lccc}
\hline Name & $\begin{array}{c}\text { OB mass } \\
(\mathrm{kg})\end{array}$ & $\begin{array}{c}\text { Cold } \\
\text { mass }(\mathrm{kg})\end{array}$ & $\begin{array}{c}\text { OB main } \\
\text { dimensions }(\mathrm{mm})\end{array}$ \\
\hline CAIN & 2 & 12 & $\varnothing 130 \times 38.1$ \\
LIRIS & 65 & 198 & $\varnothing 750 \times 185$ \\
EMCTS & 63 & variable & $\varnothing 818 \times 146$ \\
EMIR & 560 & 1,285 & $1,320 \times 1,320 \times 1,320$ \\
\hline
\end{tabular}

The supporting truss concept consists of two separate structures to withstand the forces acting in the radial and axial directions. The radial support is an arrangement of trusses loaded on a plane through the apex. The axial support is a simple system of plates directly loaded in tension or compression.
Opposite the V-truss support (as in LIRIS), for EMIR thermal contraction occurs in the direction in which the radial supports are stiff. To prevent high forces being produced during cooling, the trusses are thermally compensated. Moreover, the bending stiffness of the trusses at their ends has been reduced. Trusses are made of glass-epoxy composite G-11 (central parts) and stainless steel (flexure ends), glued together, and were manufactured in house.

The interfaces of the cryostats with the telescope frames have also evolved (see Fig. 17): from the direct and simple attachment of CAIN, to a precision alignment structure in LIRIS, to the double supporting structure of EMIR (two adapters, one for the Nasmyth rotator and other for the Nasmyth platform).

\subsection{Lens mounts}

The thermal contraction coefficients from ambient to cryogenic temperatures of lenses (typically made of fused silica, CaF2, or ZnSe) and their barrels (Al6061T6) are different: means to allow for differential contraction when cooling must be implemented, ideally preserving the alignment of the lenses at room conditions to ease alignment processes.

The problem comes with size: contraction is proportional to original dimensions, and whilst in CAIN the $\varnothing 20 \mathrm{~mm}$ camera lenses could be springloaded against fixed pads, that solution could no
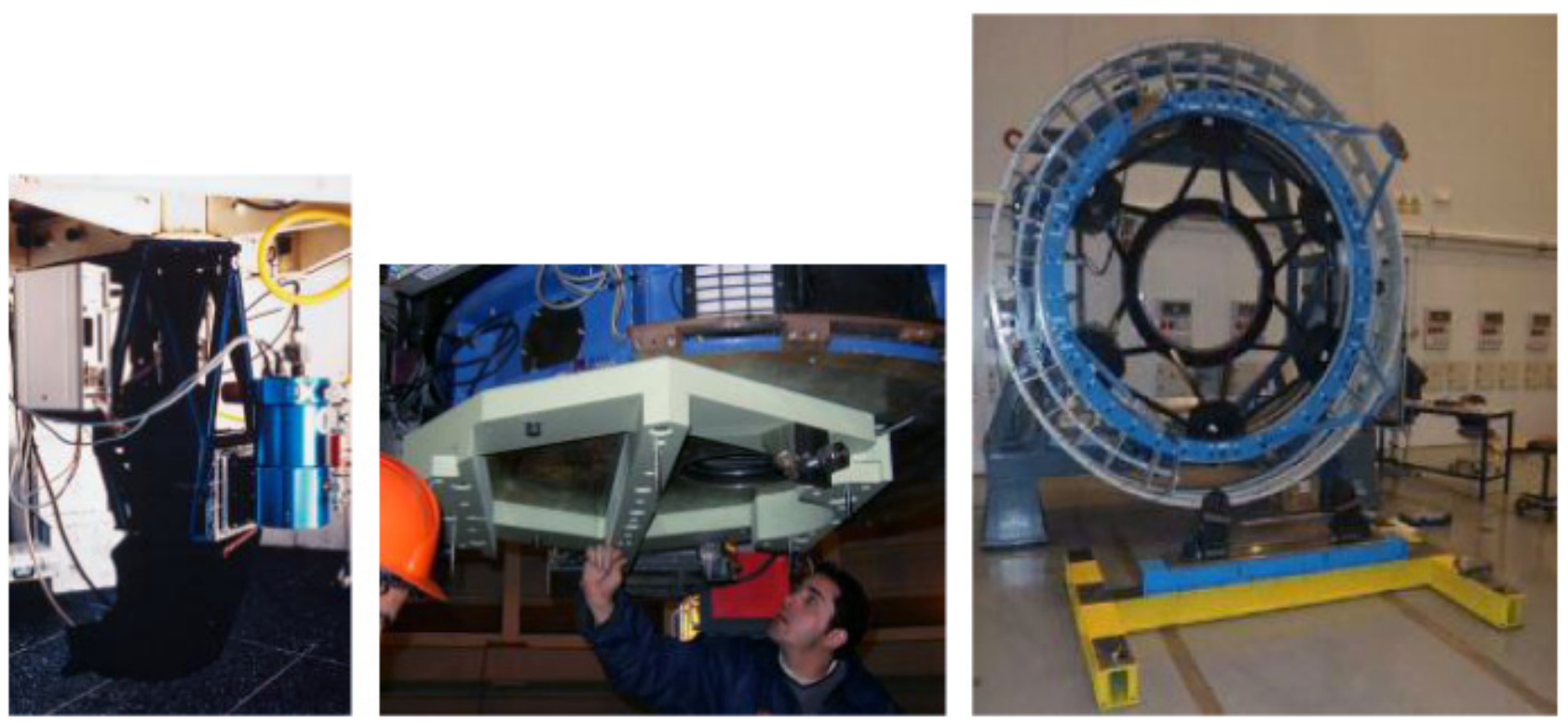

Fig. 17. CAIN, LIRIS and EMIR telescope interfaces. 
longer be applied to LIRIS (Ø100 mm) or EMIR (Ø490 mm) collimators if the alignment was to be kept both at room temperatures and at working conditions.

Two different solutions have been incorporated to cope with this limitation. First, axisymmetric flexible rings holding lenses at three points at $120^{\circ}$ are employed in the case of the LIRIS collimator (subcontracted to Janos, USA), and in the EMIR collimators and cameras (made by Seso, France except for the Collimator \#1 mount, made by the IAC). Second, the "pseudo-grism assemblies" in EMIR (made by the Laboratoire Astrophysique de Marseille, each consisting of two prisms and a grating flat) are held by mean of $120^{\circ}$ symmetrically located independent flexure "fingers."

In EMIR's Collimator \#1 "athermalized design" is achieved by the use of two fixed and one spring-loaded radial pads made out of a tailored combination of aluminum alloy and PTFE parts, which, as a whole, have the same dimensions at $20^{\circ} \mathrm{C}$ and $-196^{\circ} \mathrm{C}$. The system was designed, built and tested integrally at the IAC. This lens assembly is one of the largest cryogenic lenses mounts in the world.

Regarding filters, which have large positioning tolerances ranging in the order of millimeters, the designs have not evolved since CAIN and consist of leaf springs preloading the flats axially against the corresponding interfaces on the filter wheels, with sufficient radial clearance to allow for differential contraction.

The four mounting schemes described above can be seen in Fig. 18.

\subsection{Precision mechanisms}

The cryogenic environments inside the instruments are extreme conditions for mechanical systems. Lubrication is a major concern in components based on moving parts (bearings, precision screws, spur gears, worm gears) as conventional oils and greases (even greases designed for vacuum) will fail to keep tribological behavior under control at extremely low temperatures.

The three instruments concerned follow the policy of fully integrating the mechanisms (and the driving motors) inside the cryostats.

\subsubsection{CAIN}

The CAIN wheels (camera and filters) are moved by means of spur gears, with Vespel SP3 pinions attached directly to each motor's shaft and the wheels' aluminum alloy disks machined with

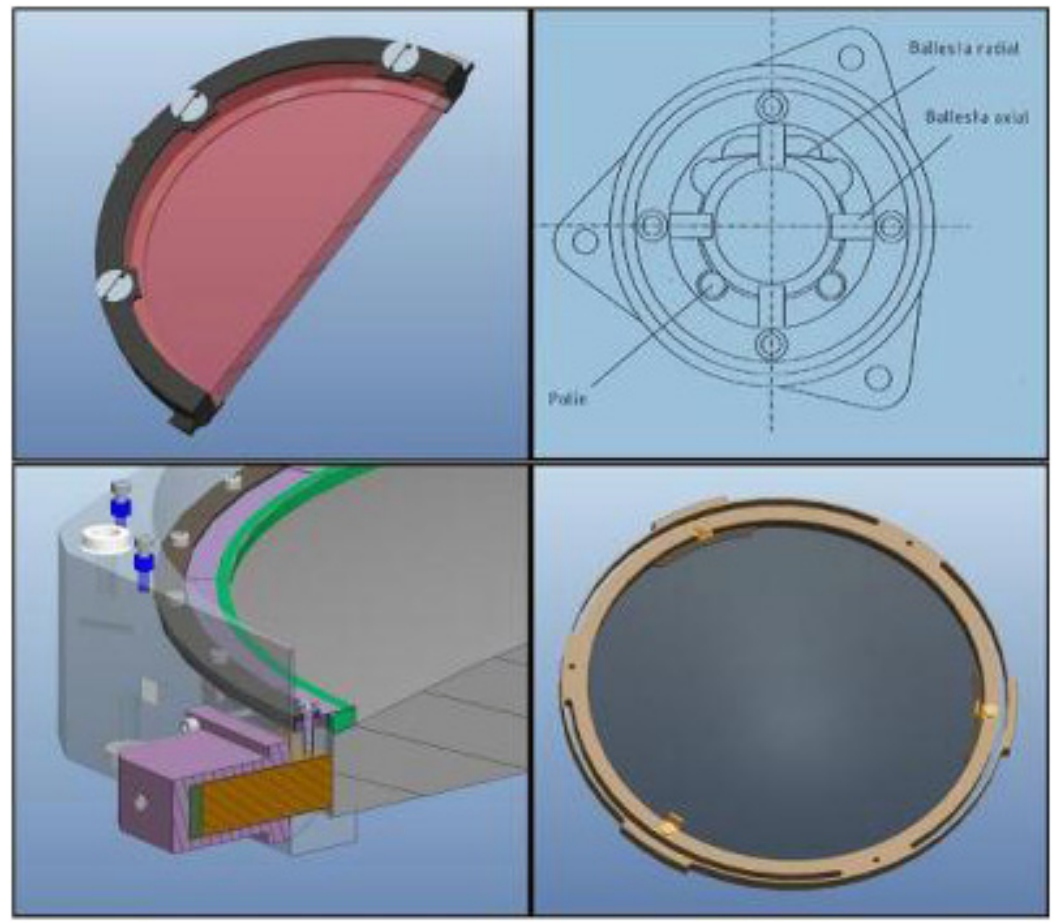

Fig. 18. Four different mounting schemes for the lenses at cryogenic temperatures (clockwise from upper left): leaf springs (filters), springs against precision positioned pads (small lenses), flexure rings (medium size), and athermalized pads (largest). 
peripheral straight teeth so as to provide the required resolution and torque reduction. The precision (only required in the camera wheel) does not rely on the motor, but a spring-loaded rocker engages v-grooves machined on the wheel instead, defining its final position. This system is practical for a small instrument such as CAIN, where alignment can be measured and corrected easily, and as dimensions are small, distortions induced by temperature gradients are easy to control. However, this solution with fixed wheel positions is not convenient for larger mechanisms and was not employed in the instruments that followed CAIN. Motors were adapted for cryogenic use by lubricating their rotor bearings with burnished $\mathrm{MoS}_{2}$, in house. Rockers were held by flex pivots, and cooling of the wheels was improved using precipitation-hardened $\mathrm{CuBe}$ bushings that also provided some degree of retaining torque to the wheels.

\subsubsection{LIRIS}

LIRIS's wheels are moved by means of worm gears actuated by stepper motors in open loop, engaging a helical gear attached to the wheel. Position relies on the final motor position, counting steps from a "home" position electronically detected by means of a microswitch. The play between the teeth in the worm-gear engagement eases the tolerancing but requires a permanent resistant torque on the wheel to hold its final position when the motor is depowered to limit heat introduced by the Joule effect in its windings. Phytron was selected as the cryogenic motor provider and has ever since been the standard at the IAC for large cryogenic steppers.

The LIRIS long-slit, multi-slit, pupil and coronography masks, which are installed in the entrance wheel and pupil wheel, were made in stainless steel AISI 304 and manufactured by wire Electric Discharge Machining (EDM), by the external companies Teckniker (Vizcaya) and Mecanizados Ginés (Logroño). Gold coating and blackening of the long-slit mask was subcontracted to INASMET (Guipuzcoa).

A good example of how much size and weight can actually complicate matters when dealing with cryogenic mechanisms, is the camera wheel in LIRIS (see Fig. 19). It is the only mechanism fully designed from scratch in house, and has essentially the same function as the camera wheel in CAIN. However the fact that the weight of the LIRIS camera is 25 times

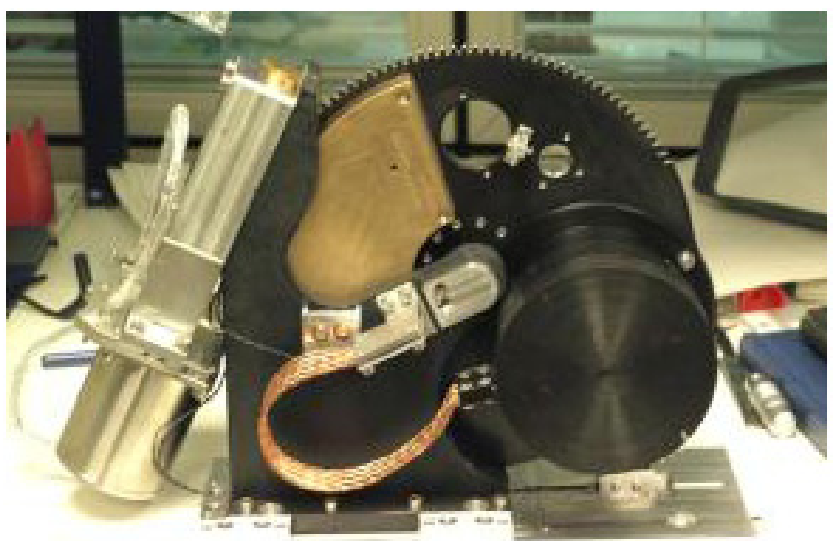

Fig. 19. LIRIS camera's wheel.

higher, together with the use of motor step counting and microswitch homing to position the wheel precisely, required several trials until a final solution to stick slip instability, braking, thermal anchoring, and microswitch activation was rounded up.

\subsubsection{EMIR}

Most EMIR mechanisms are the collaborative effort of several companies that have participated as contractors of the instrument subsystems and have contributed with their particular knowledge in the field of precision mechanisms.

One of the main design features of EMIR is the use of a cryogenic reconfigurable slit mechanism to generate a multi-slit configuration, a long slit or an imaging aperture at the telescope focal plane, the CSU. This feature will permit the instrument to remain in operational conditions for a long time and takes advantage of both a classically scheduled and a queued service observing scheme.

The CSU uses reciprocating friction pads actuated by piezoelectric stacks to displace its bars and configure slits by confronting them in pairs. The sensing of each individual bar position is made by means of capacitor sensors that, "sandwiching" a bar extension machined with windows, perceives the variations of dielectric capacity and interpolates each bar position. The technology of such an actuator solution, new to the IAC, was a contribution of Jansen Precision Engineering (Netherlands), the CSU contractor with expertise in the field of micro- and nanotechnology applications.

Another example is the detector translation unit (DTU). CSEM (Swiss Center for Electronics and Micromechanisms, Switzerland) designed and 
Table 3. Comparison between the masses and sizes of the opto-mechanical subsystems of CAIN, LIRIS, and EMIR.

\begin{tabular}{|c|c|c|c|c|}
\hline & & CAIN & LIRIS & EMIR \\
\hline \multicolumn{2}{|l|}{ Entrance window } & $\begin{array}{l}\mathrm{CaF}_{2} \text { field lens } \varnothing 47 \mathrm{~mm} \\
0.03 \mathrm{~kg}\end{array}$ & $\begin{array}{l}\text { Fused silica flat } \varnothing 120 \mathrm{~mm} \\
\quad 0.3 \mathrm{~kg}\end{array}$ & $\begin{array}{l}\text { Fused silica field lens } \\
\varnothing 500 \mathrm{~mm} 22 \mathrm{~kg}\end{array}$ \\
\hline \multicolumn{2}{|l|}{ Slits } & - & $\begin{array}{l}15(\square 60 \mathrm{~mm}) \varnothing 560 \mathrm{~mm} \\
\quad \text { wheel } 25 \mathrm{~kg}\end{array}$ & $\begin{array}{l}\text { Configurable Slit Unit } \\
\qquad(55 \times 6 \mathrm{~mm} \text { slits }) 64 \mathrm{~kg}\end{array}$ \\
\hline \multicolumn{2}{|c|}{ Collimation and folding mirrors } & - & $\begin{array}{l}\text { Integrated collimator and } \\
\text { folder mirror } \varnothing 100-50 \mathrm{~mm} \\
8 \mathrm{~kg}\end{array}$ & $\begin{array}{l}\text { 1st Collimator barrel } \\
\text { (Ø490 mm lens) } 38 \mathrm{~kg} 2 \\
\text { folder mirrors ( } \square 285 \mathrm{~mm} \\
\text { flats) } 63 \mathrm{~kg} \text { 2nd } \\
\text { Collimator barrel } \\
\text { (Ø190 mm) } 20 \mathrm{~kg}\end{array}$ \\
\hline \multicolumn{2}{|l|}{ Dispersive elements } & - & $\begin{array}{l}2 \text { Filter (22 filters } \varnothing 40 \mathrm{~mm} \\
\text { each) }+1 \text { pupils wheel }+1 \\
\text { grisms wheel } \varnothing 330 \mathrm{~mm}\end{array}$ & $\begin{array}{l}3 \text { pseudo-grisms }(9 \mathrm{~kg} \\
\text { each) Ø560 mm wheel } \\
62 \mathrm{~kg}\end{array}$ \\
\hline \multicolumn{2}{|l|}{ Filters } & $\begin{array}{l}8 \text { filters (Ø17 mm each) } \\
\varnothing 114 \mathrm{~mm} \text { wheel } 0.1 \mathrm{~kg}\end{array}$ & wheels $41 \mathrm{~kg}$ & $\begin{array}{l}14 \text { filters (Ø70 mm each) } \\
\varnothing 560 \mathrm{~mm} \text { wheel } 22 \mathrm{~kg}\end{array}$ \\
\hline \multicolumn{2}{|l|}{ Cameras } & $\begin{array}{l}\text { Wide Camera (4 lenses } \\
17-15 \mathrm{~mm}) \text { Narrow } \\
\text { Camera }(5 \text { lenses } \\
12-8 \mathrm{~mm}) 0.3 \mathrm{~kg}\end{array}$ & $\begin{array}{c}1 \text { camera }(2 \mathrm{~kg}) \text { on camera } \\
\text { wheel } \varnothing 190 \mathrm{~mm} 5 \mathrm{~kg}\end{array}$ & $\begin{array}{l}1 \text { camera }(\varnothing 150 \mathrm{~mm} \\
\text { lenses) } 28 \mathrm{~kg}\end{array}$ \\
\hline \multirow[t]{2}{*}{ Detector position } & Translation & $\begin{array}{l}\text { Manual (pads and } \\
\text { precision threaded } \\
\text { pushers) }\end{array}$ & Fixed (pads) & Motorized tripod $12 \mathrm{~kg}$ \\
\hline & Tilts & $\begin{array}{l}\text { Manual (pads and } \\
\text { precision threaded } \\
\text { pushers) }\end{array}$ & Fixed (pads) & $\begin{array}{l}\text { Nested frames with } \\
\text { flexures and precision } \\
\text { threaded pushers } 3 \mathrm{~kg}\end{array}$ \\
\hline
\end{tabular}

built for EMIR a custom tripod-like structure based on flexures that carries the detector and provides three independent degrees of freedom (translations). Each tripod leg is mounted on a linear carriage driven by a worm-nut pusher, powered by a custom stepper motor modified to be cryocompliant. These linear actuators were subcontracted to Energen (USA), as CSEM expertise was more on the side of flexures and hexapods rather than on linear actuators.

Finally, the two wheels in EMIR (pseudo-grisms and filters) were fully designed and built at the IAC. Like the camera wheel in LIRIS, the pseudogrism wheel in EMIR is a good example of how extra weight may impose challenging requirements on mechanisms. Each of the three pseudo-grisms in EMIR weighs around $8.5 \mathrm{~kg}$. The size of the wheel is close to LIRIS's slit wheel, which instead carries a total moving mass of just $3 \mathrm{~kg}$, and whose low inertia can be "neutralized" by the resisting the torque inherent in the duplexed bearing set holding the wheel disk.

EMIR's pseudo-grism wheel inertia is 60 times higher than the slit wheel in its predecessor, LIRIS, and attempts to achieve the required resistant torque by increasing the duplexed bearing set preload resulted in failure time after time. Also, the large mass involved needed extra thermal conductivity in order to achieve the required cooling times. Once the possibility of achieving both extra resistant torque and conductivity by increasing the bearing preload was given up after extensive test campaigns, efforts were directed towards the possible solution of a high conductivity, plain bearing support. Again, collaboration with an external company, in this case with expertise in the field of plasma electrolytic oxidation and the surface treatment of aluminum alloys (Keronite, UK), brought about a solution for the wheel brakes based on a technology new to the IAC.

\section{Facilities}

Since the inauguration of the present IAC Headquarters in June 1985, the evolution of the manufacturing, assembly, integration, and verification (MAIV) facilities of the IAC has been closely linked to the needs of the projects developed (in particular 


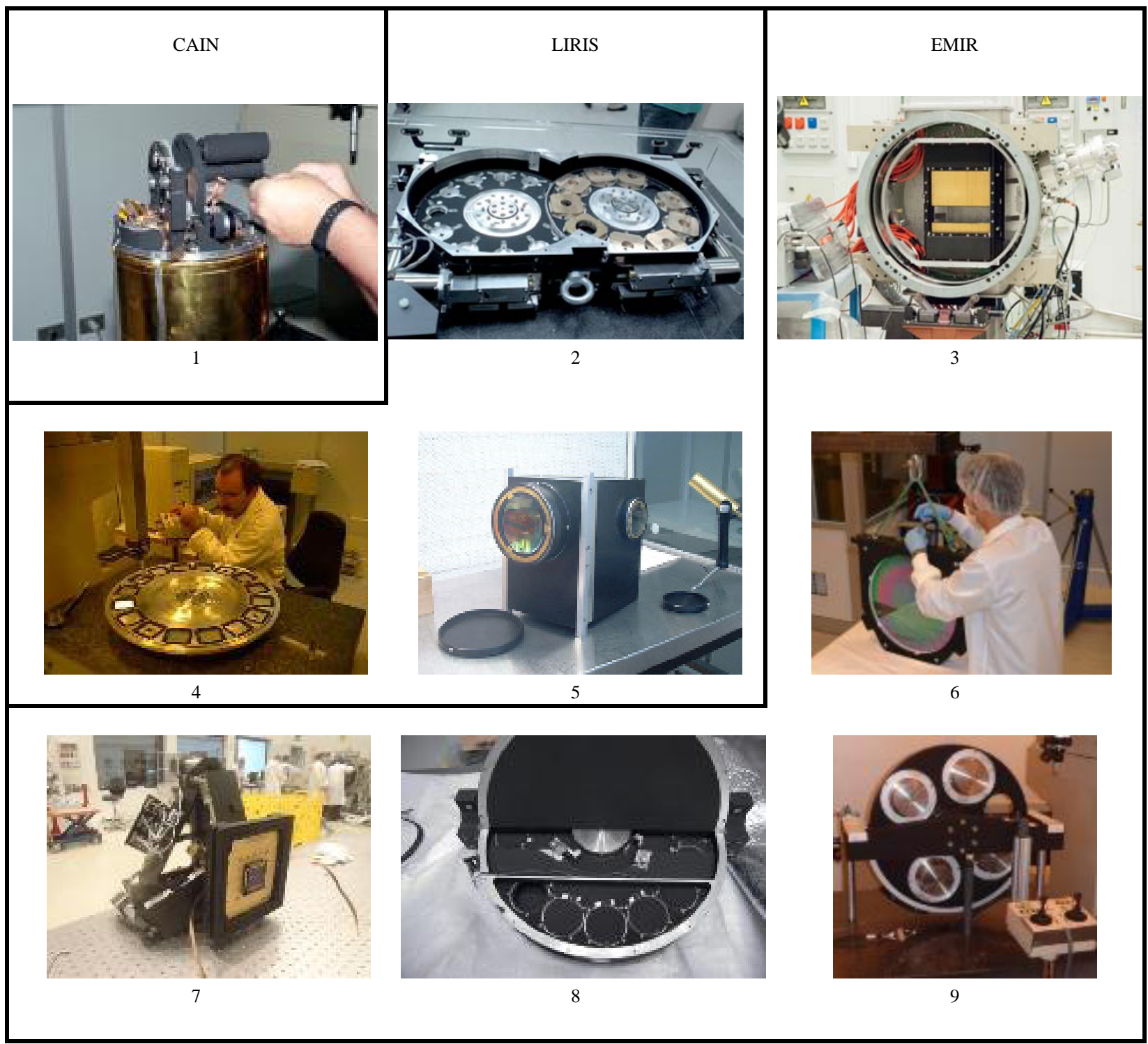

Fig. 20. CAIN (1), central wheels LIRIS (2), configurable slit unit EMIR (3), slits wheel LIRIS (4), collimator LIRIS (5), first collimator lens EMIR (6), detector traslation unit and detector assembly EMIR (7), filters unit EMIR (8), grisms unit EMIR (9).

those related to laboratories and to the mechanical workshop). Beside this, the fact of being located in a European ultra-peripheral region, where there is scant presence of industry, has influenced the need for the IAC to have its own complete facilities.

The following sections describe the more relevant facilities used in the development of the instruments described in this paper.

\subsection{Mechanics workshop}

The pace of technological progress in recent decades has brought forth many challenges for mechanical production. Highly accurate machines are now

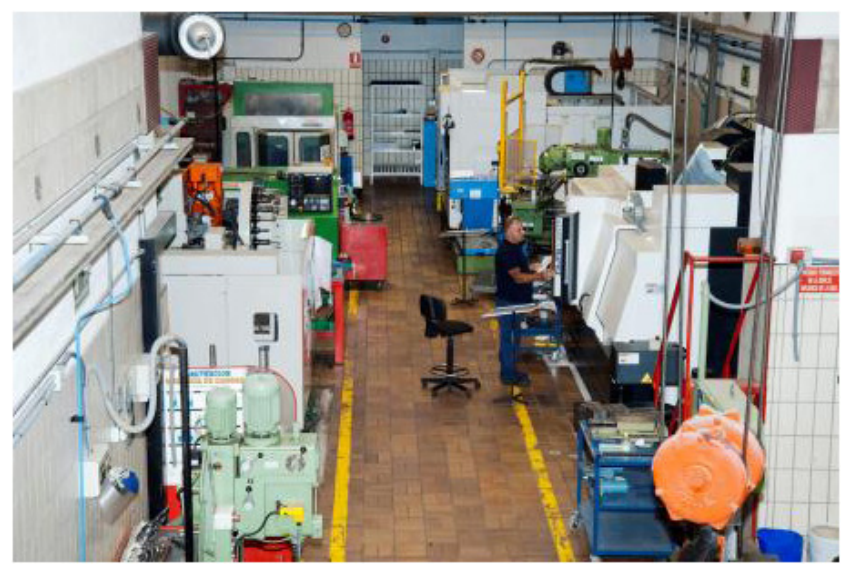

Fig. 21. The mechanics workshop. 

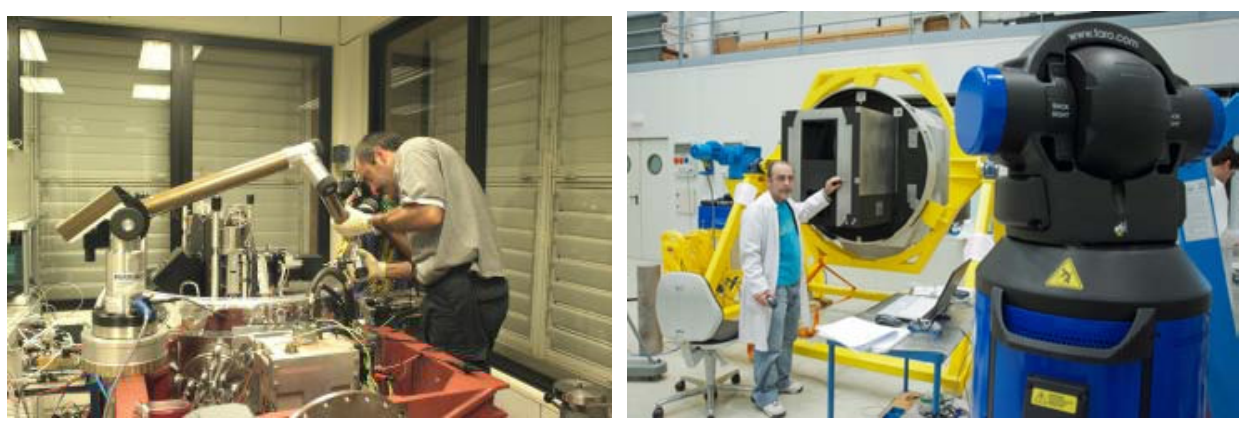

Fig. 22. IAC 3D portable measuring machines.

needed and, together with high speed machining; these are used to produce ever more complex components.

The workshop covers a floor area of $668 \mathrm{~m}^{2}$, and is divided into several areas. The machining area, with conventional and CNC lathes and both horizontal and vertical milling machines: MAZAK Quick Turn 15, Mazatech H-400, V-414, VTC 300CII, and Integrex 200-IV. The auxiliary machine area houses folding and bending machines, a bandsaw, drills, etc. The paint area has a cabinet oven and the soldering area has TIG, MIG, plasma and oxyacetylene welding capabilities. There is a heat and shot-blasting treatment area, an anodizing and ultrasonic bath area and, finally, the mechanical materials and components storage area.

The size and characteristics of the latest CNC machines installed at the IAC (MAZAK Mazatech $\mathrm{H}-400, \mathrm{~V}-414$ ), have been increased in step with the size of the instrument pieces: from 2 to 5 axis-CNC control, volumes $(\mathrm{X}, \mathrm{Y}, \mathrm{Z})$ from $560 \times 460 \times 560 \mathrm{~mm}$ to $1740 \times 760 \times 660 \mathrm{~mm}$, turning diameters from $265 \mathrm{~mm}$ to $660 \mathrm{~mm}$, and machining lengths from $500 \mathrm{~mm}$ to $995 \mathrm{~mm}$.

\subsection{Dimensional metrology laboratory}

The Dimensional Metrology Laboratory tests the components built by the IAC, as well as those manufactured by outside companies, to ensure that they conform to design specifications. The laboratory is kept at a controlled and recorded temperature and humidity environment. Besides the usual metrology equipment, in 1986 the laboratory acquired a Mitutoyo three-coordinate measuring machine with a measuring volume of $800 \mathrm{~mm} \times$ $600 \mathrm{~mm} \times 400 \mathrm{~mm}$ and 1 micron resolution with a dedicated foundation to ensure that it is not affected by external vibrations. In a similar manner to the mechanical workshop, the facilities of the metrology laboratory have expanded in accordance with the growth in size of the instrument components. So the laboratory also has two portable 3D measuring machines, a FARO arm, with a reach of $2.4 \mathrm{~m}$ in diameter and 51 microns precision, and a FARO laser tracker capable of accurately measuring coordinates within a range of $70 \mathrm{~m}$. This instrument has many features including triangulation for generating new stations and covering the envelope of any instrument to be measured, with an accuracy in the order of 20 microns +1.1 micron/meter.

\subsection{Optics laboratory}

This laboratory is used for optical testing, instrument assembly and alignment, prototype testing, and in general tasks requiring a controlled level of cleanliness, temperature, and humidity. ISOPHOT-S was the first instrument that required a clean room with a 100 class bench to avoid particle contamination of the instrument. This first facility was an ad-hoc clean room built in the Metrology Lab in 1989. In 1993 the original Optics Laboratory was enlarged, with an area of $166 \mathrm{~m}^{2}$, and

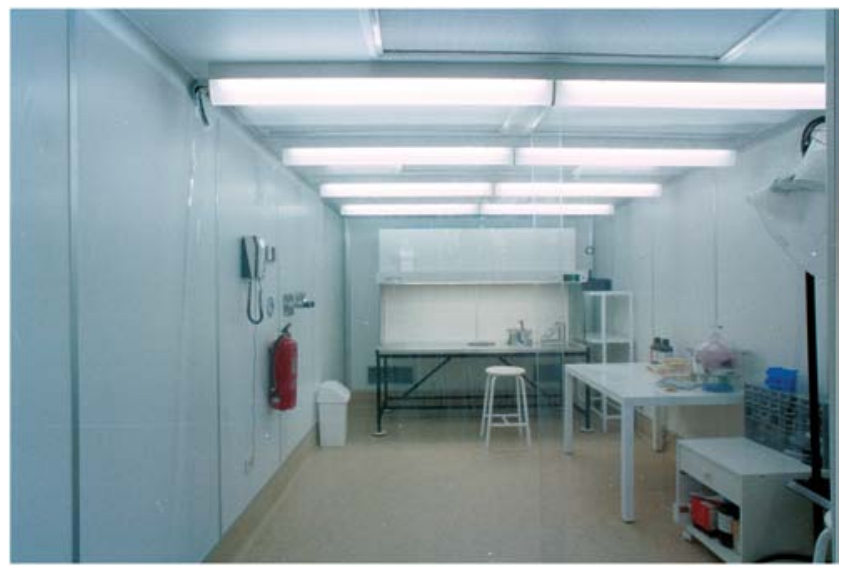

Fig. 23. Optics laboratory. 
refurbished in a 100,000 class clean room, with the 100 benches in a 1000 class clean room.

The laboratory is divided into four rooms, the access is restricted, and special clothing must be worn to maintain cleanliness. Besides the access area, there is a general area with four optical benches on a single $10 \mathrm{~m}$ long bench, with a dedicated foundation to isolate it from vibrations; two general areas with different equipment and optical tables, and a 1000 class clean work space with a 100 class bench. This last room has the highest level of cleanliness in the whole laboratory and is used to assemble space instruments, detectors, and cryogenics systems that require accurately controlled working conditions. The laboratory is equipped with a wide range of optical components such as lenses, opto-mechanical systems, lasers, optical and infrared sensors, filters and light sources, etc.

\subsection{Mechanical assembly and commissioning laboratory}

This laboratory provides general use and specialized equipment for mounting, assembling, and commissioning medium-size mechanical systems. The laboratory is equipped with a range of general purpose tests and calibrations resources, such as cryostats, a vacuum oven, high vacuum pump systems, vacuum and cryogenic hardware, data acquisition systems, torque meters, sub-micrometer calibration equipment, an ultrasonic bath, etc.

\subsection{Astronomical image and sensor laboratory}

This laboratory was built during the LIRIS AIV phase after its refurbishment to a 100,000 class clean room, since the volume of the instrument, as

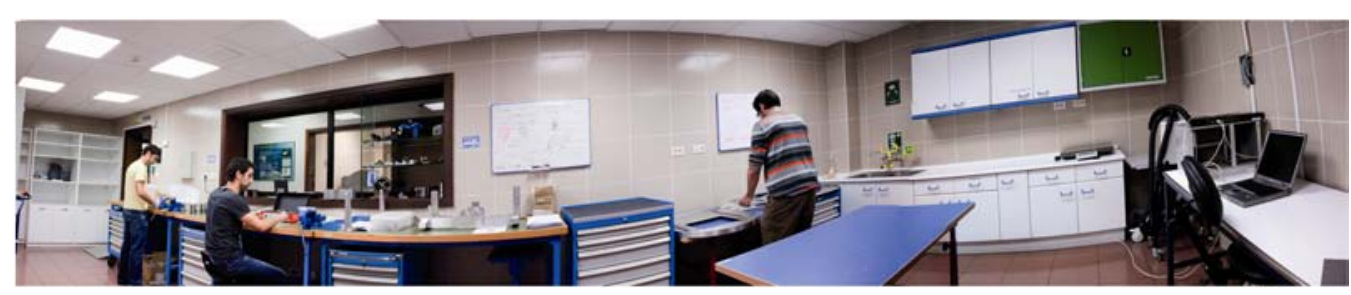

Fig. 24. Mechanical assembly and commissioning laboratory.
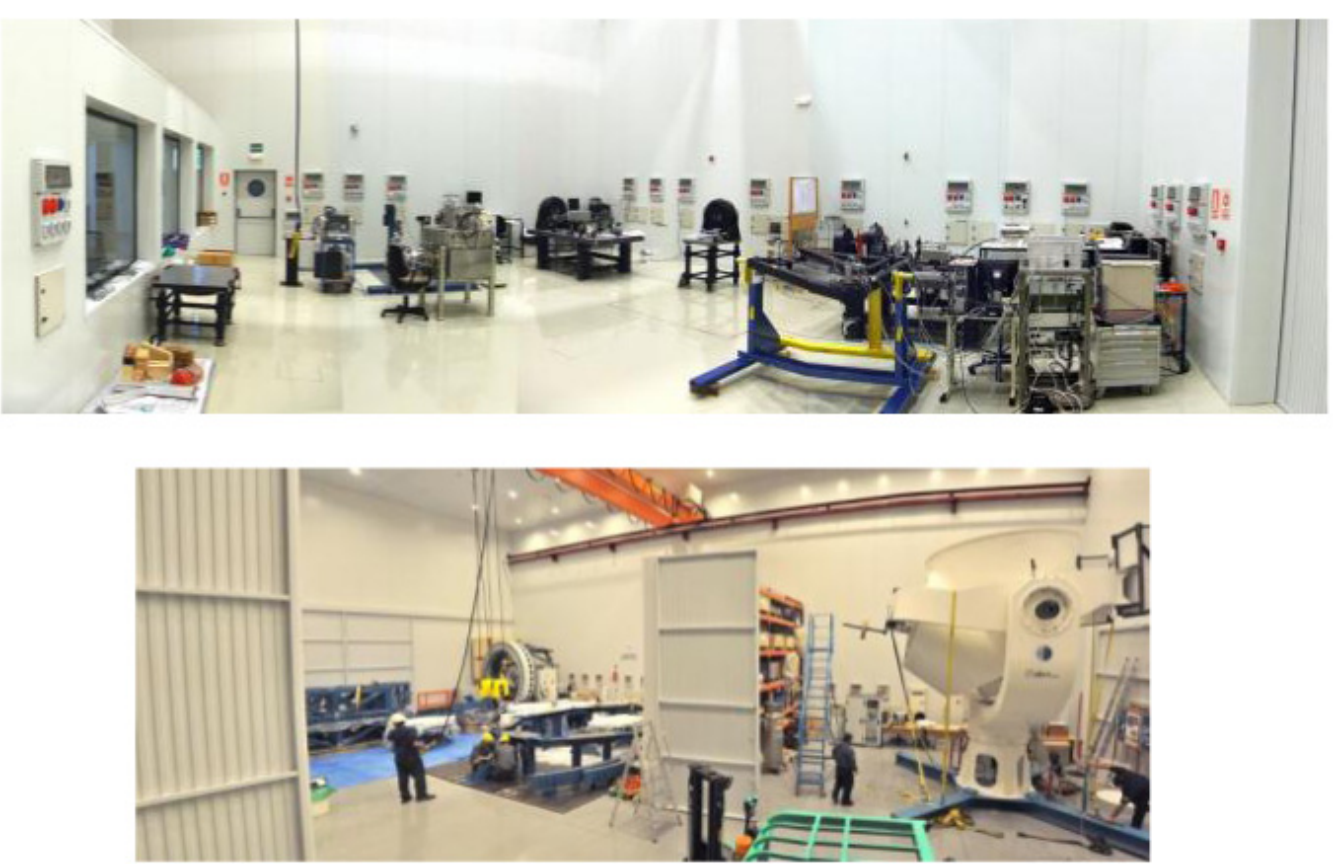

Fig. 25. AIV room. 
well as the verification facilities needed, could not be accommodated in the optical laboratory. Nowadays this laboratory, which works in both the visible and the infrared wavelength ranges, provides instruments and equipment for operating sensors in controlled conditions so that exact data about their operating parameters can be obtained for characterization.

\subsection{AIV room}

A new AIV room at the IAC was built to meet the needs of a facility large enough to assemble, integrate, and verify instruments for the new generation of large telescopes. The size and weight of the instruments for these telescopes mean that specialized handling and installation tools are needed. They need large rooms with cranes that can handle them easily, practically, and efficiently, together with a range of logistical support equipment designed for large state-of-the-art instrument development and commissioning.

The AIV room has been fully operational since the beginning of 2006 and is being used for large instrumentation projects and developments in telescopes, including the GTC and the European Extremely Large Telescope (E-ELT).

It is divided into three areas: a general use 100,000 cleanliness class laboratory, for preassembly and with dedicated foundations for optical tables with a floor area of $155 \mathrm{~m}^{2}$; the rotator area, with a GTC Nasmyth rotator simulator and two dedicated foundations, a floor area of $220 \mathrm{~m}^{2}$, refurbished into a 100,000 clean room in 2012; and a third room dedicated to receive, store or integrate equipment or instruments that do not require the clean room conditions, with an area of $125 \mathrm{~m}^{2}$. These three AIV rooms have an internal height of $10 \mathrm{~m}$.

\section{Management}

The evolution over time of the infrared projects discussed here has resulted in a gradual increase in volume and scope. We began by addressing the manufacture of CAIN, a small instrument for the TCS, breaking the project down into phases. After reaching our objectives with CAIN, we proposed the building of a larger instrument for a larger telescope, in which the IAC began its participation in the operation and where LIRIS was an important contribution. In parallel these arose the need for instrumentation for the GTC, and the IAC decided to contribute with two instruments: OSIRIS for the visible and EMIR for the infrared, both very complex instruments. The development of the two projects simultaneously and the urgency in the commissioning of OSIRIS (2008) have slowed the development of EMIR, and for this reason it has suffered a significant delay. At present EMIR is in advanced AIV phase at the IAC.

This evolution has meant that the interdisciplinary teams involved had to be increased over time and have become more specialized. External collaborators and outside manufacturers have been incorporated into the developing teams and this has allowed us to meet globally set targets.

The intention of this section is to describe the framework of available human teams, consortia established, and the timing and costs of the different instruments as an indication of the evolution of project magnitude with time.

\section{1. $C A I N$}

The first phase of the instrument ended in 1996 with the installation of CAIN, an infrared camera equipped with a $256 \times 256$ pixel Nicmos 3 detector and a filter wheel, on the TCS.

The developments required at this phase were minimal since the instrument was based on a cryostat purchased ad hoc externally and the IAC only designed and manufactured the filters wheel.

The absence of cold optics inside the cryostat prevented us from achieving the theoretical limiting magnitude of this instrument, so that phase 2, consisting of the design of a camera system that would provide a diaphragm cold pupil and a set of userselectable fields, was begun in 1997.

This phase mainly involved the optical engineering department designing optical lenses and barrels. One of the major difficulties of this phase was mounting the camera lenses, which were very small, in the barrels.

Once the camera operation was secured, the third phase improved the electronics and control software, changing mechanism drivers and the original detector controller.

The gradual development of CAIN over time is one of the main reasons behind its success in being one of the main instruments of the Carlos Sánchez Telescope today. 


\subsection{LIRIS}

After our experience with CAIN, the LIRIS feasibility study began in 1997. Lack of know-how in the development of such a large IR instrument led the IAC to contract in 1999 the conceptual design of the mechanics and the detailed design of the optics to an expert institution. This provided a solid basis from which IAC engineers and scientists developed the rest of the detailed design of the instrument.

During the detailed design review, in 2001, the AIV plan was set up, which allowed us to modify the cryostat design to include an auxiliary window that later proved to be essential in optimizing the final positioning of optics.

Three test cryostats were built to test the LIRIS subsystems before their integration. Using these cryostats, the collimator, the camera, and the slits, filter, pupil, and camera wheel mechanisms were tested under cryogenic conditions.

The engineering and the science detectors were also tested in cryogenic conditions before their integration on LIRIS, using a test cryostat.

Certainly, the use of testing cryostats was one of the keys to the successful development of LIRIS in terms of project duration and technological results.

Since LIRIS was an instrument for the WHT, and the ING, owner of the telescope, had developed INGRID, an infrared camera using the same detector and controller technology, a collaboration agreement was signed for the exploitation of LIRIS at the WHT. This collaboration between LIRIS and the ING included the use of the same detector control software and data acquisition system. This has simplified the progressive implementation of improvements on the LIRIS data acquisition system, a task carried out on a continuous basis by ING staff.

Besides operating in similar way to other IR spectrographs, LIRIS has a number of unique modes, such as coronography, multi-object spectrography, and polarimetry. Supplying the instrument with all these features from the beginning was a risk and a possible factor in the delay in its delivery. Therefore, a basic LIRIS configuration was first commissioned. This basic configuration, the imaging mode and the multi-object image mode (low resolution), left the instrument ready for the later inclusion of the high-resolution grisms, and the polarimetry and coronography modes. This wise decision allowed the LIRIS team to deliver the instrument on schedule (2003) and also permitted extra funding for the upgrades thanks to the timely generation of scientific results (the investment in high-resolution grisms, for example, was initially unaffordable).

LIRIS has been a common-use instrument at the WHT for the last 10 years and has remained the second most used WHT instrument with excellent scientific results.

\subsection{EMIR}

The development of the EMIR instrument has been a benchmark in the IR development history of infrared instrumentation at the IAC for the last 15 years. EMIR was first proposed as part of the call for proposals for first-day instruments for the GTC in 1998 and was selected in mid-1999 as the common-user IR instrument for the telescope. The conceptual design was reviewed by mid2000, but the conclusions of the review pointed out that the risks of the concept proposed were quite high. It was therefore decided to start a phase of redesign and optimization of the given concepts, especially in terms of the mechanics and optics, which ended up with a second and final Preliminary Design Review that, in mid2003, definitely launched the development of the project.

In parallel to the decision on the mechanics and optics redesign, the use of the Hawaii-II detector for EMIR had been firmly decided on since the beginning. Previous developments and experience in IR detectors at the IAC made feasible and reliable the development of a Hawaii-II control system, for which a complete characterization test system was conceived, manufactured/procured, integrated, and used with the multiplexer, engineering, and scientific grade detectors.

Due to the technical difficulties of the EMIR instrument (which can be summarized in terms of the size of components, cryogenic conditions of operation and complexity of the main subsystems, DTU, grism concept and CSU), the next step in the development of the instrument focused on the demonstration of the performance of the different subsystems by the manufacture and the testing of prototypes or demonstration models. This phase proved to be much more demanding than originally conceived and brought the project 
Table 4. Management parameters for IR instruments under consideration.

\begin{tabular}{lcccc}
\hline Name & N of institutions & $\mathrm{N}^{\circ}$ of team members & Development time & $\begin{array}{c}\text { Cost (€) (Not including } \\
\text { institutions' staff) }\end{array}$ \\
\hline CAIN (Phase 2-3) & 1 & 4 & $1994-$ August 1999 & 45,600 \\
LIRIS & 1 & 7 & Apr 1997-Mar 2004 & $1,400,000$ \\
EMIR & 4 & $17^{\mathrm{a}}$ & May 1998-December 2014 & $8,575,000^{\mathrm{b}}$ \\
\hline
\end{tabular}

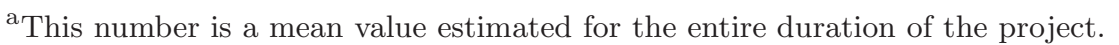

${ }^{b}$ This includes temporary manpower contracts specifically devoted to the project.

to an Advanced Design Review in March 2006. The Detailed Design and manufacturing phases of the project then started, but owing to the long delivery times of the principal subsystems (optics, DTU, CSU, and vacuum chamber), each of these was developed in different and independent ways according to different timeframes. Again, the complexity of the different subsystems and their conditions as unique prototypes for the manufacturers made the original expected development times too short. The result has been that the first integration of the instrument at a system level could not take place until the delivery of the vacuum chamber in May 2012.

At present, one year later, the EMIR project team is deeply involved in the integration and test phases of the whole system after delivery of the last mechanism, the CSU, in June 2013. It is planned to install EMIR on the GTC by mid-2015.

The EMIR project has been organized as a consortium comprising the following institutions:

- The IAC (the chief institution and main contributor)

- The Universidad Complutense de Madrid (UCM, Spain)

- The Laboratoire Astrophysique de ToulouseTarbes (LATT, France)

- The Laboratoire d'Astrophysique de Marseille (LAM, France)

All the engineering development areas, management, optics, mechanics, electronics, and software have been managed by the EMIR engineering team at the IAC, with a mean total number of members of about 17 people throughout the duration of the project.

Table 4 gives a summary of the principal parameters related to the development and management of the three IR instruments considered.

\section{Acknowledgments}

We dedicate this paper to the memory of those of our colleagues who are no longer with us.

The authors would like to dedicate this paper to people of all the projects teams who have participated in these developments.

\section{References}

Álvarez Martín, P. et al., 1995, The ORM 8-m Telescope: Proposal, La Laguna: Gran Telescopio Canarias.

Álvarez Martín, P. et al., 1997, Gran Telescopio CANARIAS: Conceptual Design, La Laguna: Gran Telescopio Canarias.

Barlow, J. V., 1975, The Astronomical Telescope, London: Wykeham Publications, pp. 163-164.

Dominguez-Tagle, C., Sanchez, V., Lopez, R., Acosta, J. \& Manchado, A., 2001, Multi-slit mask manufacturing for LIRIS, SAIT, AstroTech Journal, 4, 1.

Eikenberry, S. S., Bennett, J. G., Chinn, B. et al., 2012, MIRADAS for the Gran Telescopio Canarias: System overview, SPIE Proceedings, Vol. 8446, doi:10.1117/12. 925686.

Fuentes, F. J., Gonzalez, J. P. P., Cadavid, E. et al., 1995, First results of the Instituto de Astrofisica de Canarias infrared camera, SPIE Proceedings, Vol. 2475, doi:10.1117/12.211257.

Garzón, F., Abreu, D., Barrera, S. et al., 2006, Ground-based and Airborne Instrumentation for Astronomy 2006.6269, p. 626918-1.

Garzón, F., Abreu, D., Barrera, S. et al., 2006, SPIE Proceedings, Vol. 6269, doi:10.1117/ 12.671302.

Garzón, F., Atad-Ettedgui, E., Hammersley, P. et al., 2006, SMART-MOS: A NIR imager-MOS for the ELT, SPIE Proceedings, Vol. 6273, doi:10.1117/ 12.671675.

Lemke, D., Garzon, F., Gemuend, H.-P. et al., 1993, ISOPHOT: Far-infrared imaging, polarimetry, and spectrophotometry on Infrared Space Observatory, This paper was published in SPIE Proceedings, Vol. 2019, doi: $10.1117 / 12.157847$.

Manchado-Torres, A., Fuentes, F. J., Prada, F. et al., 1998, LIRIS: A long-slit intermediate-resolution infrared spectrograph for the WHT, Proc. SPIE, 4841, Published: doi:10.1117/12.317270.

Ring, J., 1968, "Infrared Astronomy", Imperial Lecture 4 June 1968 (Imperial College of Science and Technology, London). 
Sánchez, B., Acosta, J. A., Álvarez, L. C. et al., 2012, Current status of FRIDA: Diffraction limited NIR instrument for the GTC, SPIE Proceedings, Vol. 8446, doi:10.1117/12. 926499.

Sánchez, F. \& Wall, J. V., 1991, Astrophy. Lett. \& Comm., 28, 47.
Selby, M. J., "Infrared observations at Tenerife" I00/1973 Royal Observatory of Edinburgh The 150th Anniv. of the Roy. Obs. Edinburgh and Infrared Astronomy, pp. 48-54 (SEE N76-12899 03-89). 